\title{
Nutrient cycling and self-regulation determine food web stability
}

\author{
Kevin Theis ${ }^{1, *}$, Pierre Quévreux ${ }^{1, *}$, and Michel Loreau ${ }^{1}$ \\ ${ }^{1}$ Theoretical and Experimental Ecology Station, UPR 2001, CNRS, 09200 Moulis, France \\ *authors contributed equally
}

\begin{abstract}
To understand ecosystems, an integrative approach combining functional ecology and community ecology is required. Nutrient cycling is a good example since it links each organism to the major flows of materials in ecosystems. Together with the demographic processes governing the mortality of organisms (and hence their nutrient losses) such as self-regulation, nutrient recycling has a major impact on ecosystem dynamics and stability. By considering stochastic perturbations in the vicinity of the equilibrium affecting the top species in a food chain, we assessed stability based on the temporal variability in the different compartments of the food chain for different recycling efficiencies and self-regulation intensities. Our results show that nutrient cycling always has a destabilising effect on perturbed species, while lower trophic levels are stabilised or destabilised depending on their trophic distance from the perturbed species. Thus, for species at odd distances from the top species, nutrient cycling is stabilising, whereas for species at even distances, nutrient cycling is destabilising. Self-regulation generally stabilises systems, unless its effects are too strong. Finally, nutrient cycling and self-regulation have opposite effects because nutrient cycling dampens the stabilising effect of self-regulation. Considering these two phenomena together is necessary to assess the effects of perturbations on species dynamics and thus to understand the overall response of ecosystems in the context of global changes.
\end{abstract}

\section{Key words}

nutrients, food chain, invariability, stochastic perturbations, positive feedbacks, bottom-up, top-down

\section{Introduction}

The study of ecosystems in light of functional ecology and community ecology frameworks has led to major research progress in recent decades (DeAngelis, 1992; Loreau, 2010; McCann, 2012). In fact, these two frameworks taken together describe how mechanisms at the individual level (i.e., growth rates and mortality rates) can impact the functioning of an entire ecosystem. For instance, nutrient cycling, which is the loss of nutrients by organisms (e.g., excretion, faeces and dead bodies) that can be reabsorbed by primary producers, perfectly illustrates the productive combination of ecosystem functioning and community ecology. In fact, nutrient cycling links each organism to the base of the food chain, thus creating feedback loops likely to affect the global dynamics of ecosystems (Ulanowicz, 1990; Loreau, 1998; Loreau, 2010; Veldhuis et al., 2018a). Nutrient cycling can deeply alter food web dynamics, which was not considered in previous studies using community models only. For example, Leroux and Loreau (2010) showed that

corresponding author: pierre.quevreux@cri-paris.org nutrient cycling alters the mechanisms causing trophic cascades by increasing primary producer biomass. The importance of nutrient cycling in ecosystem stability has received specific attention; nevertheless, it has led to contrasting results depending on the definition of stability considered in different studies (O'Neill, 1976; DeAngelis, 1980, 1992; DeAngelis et al., 1989; Loreau, 1994, 2010; McCann, 2012; Neutel and Thorne, 2014; Quévreux et al., 2021b).

The notion of stability is subject of intense debate in ecology (Grimm and Wissel, 1997; McCann, 2000), and its various definitions do not measure the same features of ecosystem dynamics (Arnoldi et al., 2016). Studies considering asymptotic resilience, i.e. the rate at which a system returns to equilibrium after a perturbation in a very long run, as measured by the lead eigenvalue of the Jacobian matrix of the system, showed that stability decreases when nutrient recycling becomes more efficient (DeAngelis, 1980; Nakajima and DeAngelis, 1989; DeAngelis et al., 1989; DeAngelis, 1992; Loreau, 1994). However, Loreau (1994) tempered this conclusion by showing that nutrient recycling efficiency increases resistance (defined as the magnitude of the deviation of the system from equilibrium after being perturbed). Thus, these mea- 


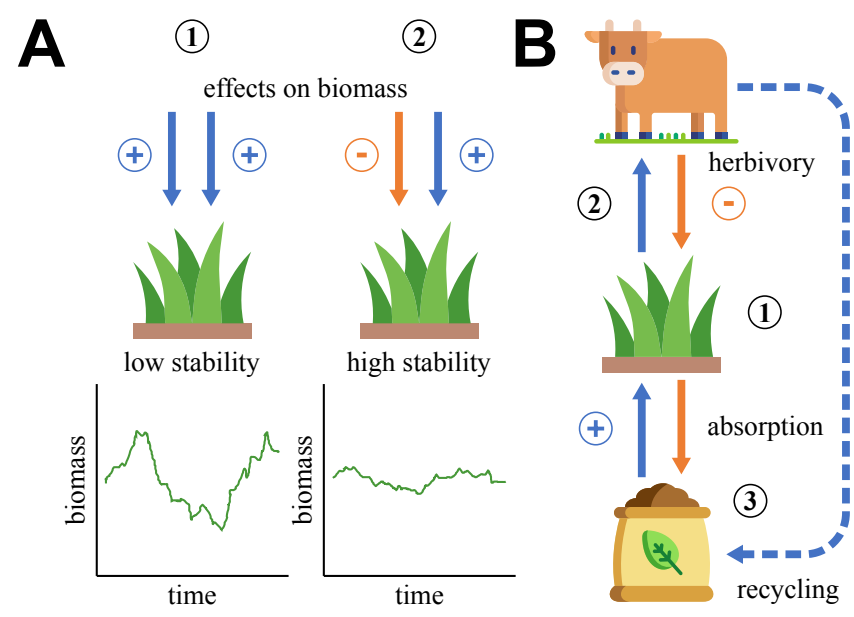

Figure 1: Expected effects on biomass of nutrient cycling feedback loops in a food chain with two trophic levels. A) Net effects on biomass should translate into stability. (1) Effects with the same direction add up and lead to large variations in biomass, thus decreasing stability. (2) Effects with opposite directions cancel each other and lead to small variations in biomass, thus increasing stability. B) In a food chain, this should lead to the stabilisation or the destabilisation of the different trophic levels. Orange arrows represent the negative effect of consumers on their resource while blue solid arrows represent the positive effect of resources on their consumer. Blue dashed arrows represent the positive effect of nutrients recycled by organisms on mineral nutrients. (1) Nutrient cycling generates a positive feedback of herbivores on primary producers due to the increased nutrient availability. This positive effect should offset the negative effect of herbivory and stabilise the dynamics of primary producer biomass. (2) Nutrient cycling generates a positive feedback of herbivores on themselves that should amplify the variations of herbivore biomass and destabilise its dynamics. (3) In a similar way, herbivores have a positive indirect effect on mineral nutrients that adds up to the positive effect of nutrient cycling feedback loop, thus leading to a destabilisation of mineral nutrients.

sures describe different facets of stability, and each of them has intrinsic limitations. In particular, asymptotic resilience has often been used to describe the stability of the entire system but Haegeman et al. (2016) and Arnoldi et al. (2018) demonstrated that it is actually driven by rare species and ignores abundant species, which contribute most to total biomass. Hence, asymptotic resilience is not a good integrative metric of ecosystem stability. Other measures, such as species persistence (the fraction of surviving species after a given time) and temporal variability (the coefficient of variation of biomass), describe the response of each species (Brose et al., 2006; Heckmann et al., 2012; Shanafelt and Loreau, 2018), and provide more insight into stability at different scales (from population to ecosystem, see Haegeman et al., 2016). Moreover, temporal variability is often used in empirical studies (Tilman et al., 2006; Gross et al., 2014; Rakowski et al., 2019 ); thus, using this measure of stability in mathematical models strengthens the relevance of theoretical results for empirical ecology. Quévreux et al. (2021b), who considered a food web model including up to 50 species and a maximum of four trophic levels, showed with these two measures that nutrient cycling affects food web stability mainly through its enrichment effect. At low nutrient inputs, nutrients recycled by all the organisms of the food web sustain a higher carnivore or consumer biomass and increase species persistence (stabilising effect). In contrast, at high nutrient inputs, recycled nutrients promote the paradox of enrichment and thus have a destabilising effect.

In addition to this enrichment effect, nutrient cycling creates links between each trophic level and mineral nutrients (Vanni, 2002; Veldhuis et al., 2018a,b), thus generating feedback loops whose consequences have only been explored by a few studies. For example, Brown et al. (2004) found that such feedback loops can delay or even prevent the appearance of limit cycles due to increased external nutrient inputs. In Quévreux et al. (2021b), the effects of these feedback loops, despite being slight, depend on the considered trophic level since they are stabilising for consumers and destabilising for primary producers. In our study, we expect the feedback loops generated by nutrient cycling to interact with the effect of predation according to the trophic position of each species (Fig. 1A). Shanafelt and Loreau (2018) demonstrated the existence of trophic cascades in the stability of the various trophic levels, i.e. trophic levels at even distance from the top are the most stable while trophic levels at odd distance, which experience a strong top-down control, are the least stable. Hence, we expect nutrient recycled by predators to generate an indirect, positive effect on prey that would offset the negative direct effect of predation and thus to stabilise prey dynamics (Fig. 1B (1)). However, this positive effect is also expected to amplify and destabilise the existing trophic dynamics of predators and compartments at odd trophic distance from the considered predators (Fig. 1B (2) and (3)).

These mechanisms governing stability should be modulated by demographic processes affecting both nutrient cycling and top-down control such as selfregulation, which summarises intraspecific interactions limiting population growth (e.g., territoriality and disease transmission, shading). By increasing mortality, self-regulation increases the quantity of nutrients recycled by organisms. In addition, self-regulation stabilises population dynamics by reducing interspecific interaction strength relative to intraspecific interactions, as supported by numerous theoretical and empirical studies (Yodzis, 1981; Begon et al., 1986a,b; Sterner et al., 1997; Moore and Ruiter, 2012; Barabás et al., 2017; Barbier and Loreau, 
2019; Picoche and Barraquand, 2019, 2020). Thus, we expect self-regulation to amplify the stabilising or destabilising effects of nutrient cycling described earlier by increasing the positive bottom-up effect of nutrient cycling and decreasing the negative top-down effect of predators on prey.

To explore these mechanisms, we extend the model developed by Shanafelt and Loreau (2018) by implementing nutrient cycling and measured its response to stochastic perturbations to assess food chain stability. This approach, which is based on stochastic perturbations of the system in the vicinity of equilibrium, considers biomass variability as a measure of stability and enables us to track the response of each compartment of the system (Shanafelt and Loreau, 2018; Arnoldi et al., 2019; Barbier and Loreau, 2019). We first explore the effect of nutrient cycling on stability by varying recycling efficiency (i.e., the fraction of excreted nutrients that returns to the mineral pool) by maintaining nutrient availability constant to disentangle feedback effects from enrichment effects according to Quévreux et al. (2021b) and McCann (2012). Then, we detail how these effects are modulated by self-regulation, which partially controls the losses of nutrients by organisms. Finally, we vary food chain length to understand how predation and trophic position influence the effects of nutrient cycling on food chain stability.

\section{Methods}

\section{General description of the model}

We extend the model developed by Shanafelt and Loreau (2018), which consists of a food chain sustained by mineral nutrients, by adding nutrient cycling, which returns a fraction $\lambda$ of nutrients lost by organisms (mortality, excretion, and inefficient feeding) to the mineral nutrient compartment (Fig. 2). The fraction $1-\lambda$ leaves the ecosystem, thus classic food chain models from the community ecology framework without nutrient cycling are completely open (i.e. $\lambda=0$ ), since all the biomass lost by organisms is also lost by the ecosystem. This approach is the simplest way to model nutrient cycling (DeAngelis, 1980; Loreau, 1994; Leroux and Loreau, 2010), and is relevant here as we aim to capture the fundamental effects of recycling feedback loops on food web dynamics (Brown et al., 2004). However, more realistic models exist that involve detritus and decomposers for instance (DeAngelis et al., 1989; Loreau, 1995; Zou et al., 2016). The dynamics of mineral nutrients and species biomass are described in Box 1 and the parameters used in the model are listed in Table 1.

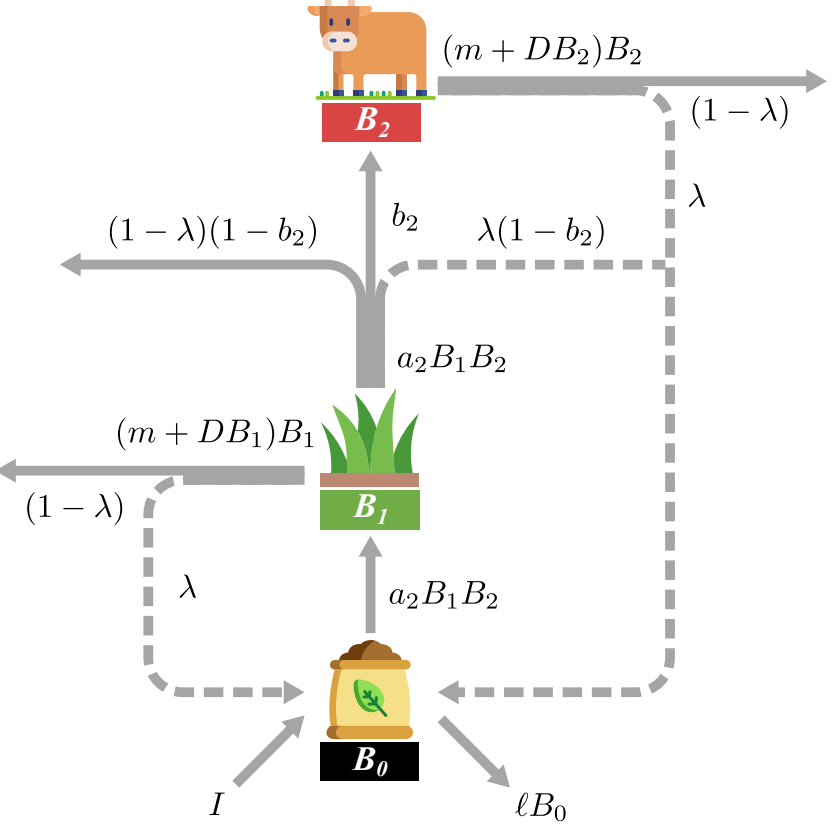

Figure 2: General food chain model with two trophic levels (TL2): mineral nutrients $B_{0}$, primary producers $B_{1}$ and herbivores $B_{2} . I$ is the external nutrient input and $\ell$ is the leaching rate. $b_{i}$ is the biomass conversion efficiency, $a_{i}$ is the predation rate of species $i$ on species $i-1, m$ is the loss rate due to death, respiration or excretion and $D$ is the self-regulation. The flows of nutrients governed by these parameters are represented by solid arrows. The recycling efficiency $\lambda$ is defined as the fraction of lost nutrients (due to mortality, self-regulation and inefficient feeding) returning to the mineral nutrient compartment. These flows of recycled nutrients are represented by dashed arrows.

\section{Stochastic perturbations}

Variability is generated by small stochastic perturbations affecting the system in the vicinity of the equilibrium according to the following stochastic differential equations:

$d B_{i}=f_{i}\left(B_{1}, \ldots, B_{n}\right) d t+\sigma_{i} B_{i} d W_{i}$

$f_{i}\left(B_{1}, \ldots, B_{n}\right)$ represents the deterministic part of species $i$ dynamics depending on the biomass of the $n$ species present in the ecosystem (described by equation (1)). The right part of the equation represents the stochastic perturbations defined by their standard deviation $\sigma_{i}$, a white noise $d W_{i}$ of mean 0 and variance 1 and scale with species $i$ biomass $B_{i}$ (Wang et al., 2015; Arnoldi et al., 2016; Shanafelt and Loreau, 2018).

\section{Assessing stability}

We measure stability by invariability $S_{i}$, which is defined as the inverse of the coefficient of variation of species $i$ biomass $\left(C V_{i}\right)$, i.e. the ratio of the mean biomass $B_{i}^{*}$ to its standard deviation $\sqrt{w_{i}}$ (Shanafelt and Loreau, 2018). Note that mean biomass is here 
Box 1: Food chain model

$$
\begin{aligned}
\frac{d B_{0}}{d t} & =I-\ell B_{0}-a_{1} B_{0} B_{1}+\sum_{i=1}^{n} \lambda\left(m+D B_{i}\right) B_{i}+\sum_{i=2}^{n} \lambda\left(1-b_{i}\right) a_{i} B_{i-1} B_{i} \\
\frac{d B_{1}}{d t} & =B_{1}\left(a_{1} B_{0}-a_{2} B_{2}-m-D B_{1}\right) \\
\frac{d B_{i}}{d t} & =B_{i}\left(b_{i} a_{i} B_{i-1}-a_{i+1} B_{i+1}-m-D B_{i}\right)
\end{aligned}
$$

$B_{i}$ is the biomass of trophic level $i$, where $B_{0}$ corresponds to mineral nutrients, $B_{1}$ to primary producers, $B_{2}$ to herbivores and $B_{i}(i>2)$ to carnivores. We consider Lotka-Volterra trophic interactions with $b_{i}$ the biomass conversion efficiency (we assume that primary producers convert all the absorbed mineral nutrients into biomass) and $a_{i}$ the predation rate of specie $i$ on specie $i-1$. Species are subject to losses $m$ due to death, respiration or excretion and self-regulation $D$ (negative intraspecific interactions). A fraction $\lambda$ of the biomass lost by organisms (losses $m$ and self-regulation $D$ ) and inefficient feeding (fraction $1-b_{i}$ of the ingested biomass) is recycled by returning to the mineral nutrient compartment. Finally, $I$ represents the external nutrient inputs and $\ell$ represents the leaching rate (nutrients running off the ecosystem). Parameters and their values are detailed in Table 1.

equal to the biomass at equilibrium since we consider small perturbations in the vicinity of the equilibrium. Thus, the higher invariability, the lower the temporal variability of biomass and the higher the stability of the biomass dynamics of the considered species.

$S_{i}=\frac{1}{C V_{i}}=\frac{B_{i}^{*}}{\sqrt{w_{i}}}$

Biomasses at equilibrium $B_{i}^{*}$ are calculated by numerical integration of equation (1) with the odeint function from the SciPy package (version 1.5.2) of the Python programming language for 4,000 to 1,000,000 time units, which are enough to reach equilibrium. Initial biomasses are obtained by the analytic resolution of the system for $\lambda=1$ and $D=0$ or $D>0$.

Variances $w_{i}$ are the diagonal elements of the variancecovariance matrix $C^{*}$ of the system obtained by solving the Lyapunov equation (Arnold, 1974; Wang et al., 2015; Arnoldi et al., 2016; Shanafelt and Loreau, 2018):

$J C^{*}+C^{*} J^{T}+T V_{E} T^{T}=0$

$J$ is the Jacobian matrix, $V_{E}$ is the covariance matrix of perturbations and $T$ describes how perturbations affect the system. (Matrices are detailed in section S11 in the supporting information and see Quévreux et al., 2021a for a detailed description of the Lyapunov equation).

\section{Addressing nutrient availability}

We aimed to study the feedback loops generated by nutrient cycling, but nutrient cycling also has an enrichment effect that must be carefully taken into account. First, the impact of nutrient cycling depends on the contribution of recycled nutrients compared to that of external nutrient inputs. Thus, in an ecosystem with

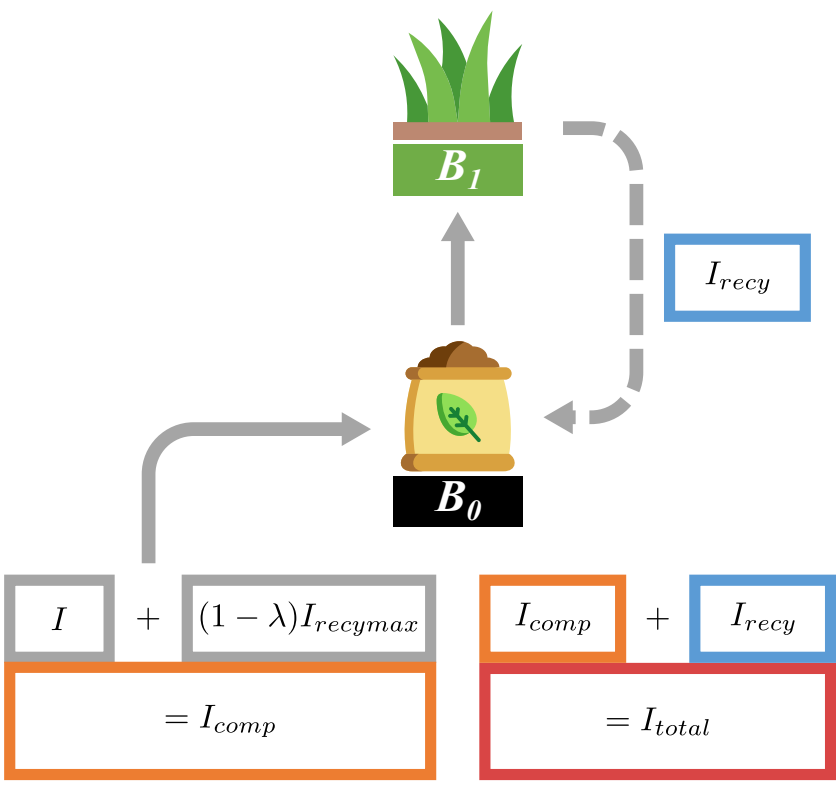

Figure 3: Schematic summarising the different flows of nutrients in the ecosystem. $B_{0}$ is the mineral nutrient compartment and $B_{1}$ is the primary producer biomass. $I$ is the basal external input, $I_{\text {recy }}$ is the quantity of recycled nutrients and $I_{\text {recymax }}$ is its value when $\lambda=1$. $I_{\text {comp }}$ is the additional external input offsetting fraction $1-\lambda$ of nonrecycled nutrients. Thus, $I_{\text {total }}$ is constant regardless of the value of $\lambda$.

low external inputs, recycling would contribute significantly to the supply of the mineral nutrient compartment. This relation is summarised by the ratio of external inputs $I$ to internal inputs of recycled nutrients $I_{\text {recymax }}$ (i.e., maximum quantity of recycled nutrients when $\lambda=1)$. Thus, $I / I_{\text {recymax }}$ is maintained between 0.1 and 1 to ensure a significant contribution of nutrient cycling to the overall dynamics of our ecosystem 
Table 1: Parameters derived from Shanafelt and Loreau (2018).

\begin{tabular}{cllc}
\hline Parameters & \multicolumn{1}{c}{ Interpretation } & \multicolumn{1}{c}{ Units } & Values \\
\hline$I$ & external nutrient inputs & ${\text { density } \times \text { time }^{-1}}^{-1}$ & $\{0.05,0.1\}$ \\
$\ell$ & leaching rate & time $^{-1}$ & 0.1 \\
$D$ & density-dependent mortality (self-regulation) & density $^{-1} \times$ time $^{-1}$ & {$[0,1]$} \\
$\lambda$ & recycling efficiency & dimensionless $^{-1}$ & {$[0,1]$} \\
$a_{1}$ & attack rate of $B_{1}$ on $B_{0}$ & density $^{-1} \times$ time $^{-1}$ & 2 \\
$a_{2}$ & attack rate of $B_{2}$ on $B_{1}$ & density $^{-1} \times$ time $^{-1}$ & $\{0.4,0.401\}$ \\
$a_{3}$ & attack rate of $B_{3}$ on $B_{2}$ & density $^{-1} \times$ time $^{-1}$ & 0.5 \\
$b_{2}$ & biomass conversion efficiency from $B_{1}$ to $B_{2}$ & dimensionless & 0.5 \\
$b_{3}$ & biomass conversion efficiency from $B_{2}$ to $B_{3}$ & dimensionless $^{-1}$ & 0.8 \\
$m$ & mortality rate & time & 0.1 \\
$I / I_{\text {recymax }}$ & ratio of external input to input by nutrient cycling & dimensionless & ] $0,1]$ \\
\hline
\end{tabular}

model. We do not constrain $I / I_{\text {recymax }}$ to a fixed value because we already adjust the values of $I$ and $D$ (see equations (8) and (9) in the supporting information). Second, varying recycling efficiency $\lambda$ simultaneously increases feedback and enrichment effects. To disentangle these two effects, we offset fraction $1-\lambda$ of nutrients lost by the system due to inefficient recycling by an additional external nutrient input, as in Quévreux et al. (2021b) (Fig. 3). This modified external input $I_{\text {comp }}$ is defined by:

$I_{\text {comp }}=I+(1-\lambda) I_{\text {recymax }}$

Thus, we maintain a constant nutrient availability regardless of the value of $\lambda$, which ensures similar biomasses and species persistence for all values of $\lambda$.

\section{Results}

\section{Recycling efficiency}

To understand the influence of nutrient cycling on food chain stability, we first consider a nutrient-primary producer system where only primary producers are perturbed. We choose this simple setup because it results in an easier mathematical analysis, and models with a longer food chain length are studied in Figs. S22 and S2-3 in the supporting information.

First, the invariability in the primary producers $\left(B_{1}\right)$ decreases when the recycling efficiency $\lambda$ increases regardless of the value of the self-regulation coefficients $D$. This decrease is due to a stronger increase in standard deviation $\sigma_{1}$ than in equilibrium biomass $B_{1}^{*}$ (Fig. 4). In the same way, the invariability in the mineral nutrients $\left(B_{0}\right)$ is driven by the variation in the standard deviation but also depends strongly on the self-regulation coefficient $D$. In fact, for $D \neq 0$, we observe a maximum invariability at $\lambda=0.53$ due to a minimum standard deviation.

These variations can be fully explained analytically by the values of the term $J_{01}$ of the Jacobian matrix which represents the direct effect of $B_{1}$ on $B_{0}$ (see equation (12) in the supporting information). This direct effect has two components with opposite effects: the absorption of $B_{0}$ by $B_{1}(1)$ and the recycling effect of $B_{1}(2)$ (Fig. 5A). For low values of $\lambda$, the negative effect of absorption overwhelms the positive effect of recycling and perturbations are mainly transmitted from $B_{1}$ to $B_{0}$ through absorption, thus leading to a negative overall effect (Fig. 5B). As the recycling efficiency $\lambda$ increases, the recycling effect becomes stronger and finally totally offsets the absorption effect. This represents a situation where primary producers have a null net effect on nutrients and thus corresponds to the maximum invariability observed in Fig. 4. Then, for high values of $\lambda$, perturbations are mainly transmitted by nutrient cycling. However, this pattern does not hold when the self-regulation coefficient $D$ is null because the recycling effect is weak to offset the absorption effect (Fig. 5B).

We obtain similar results for other food chain lengths since the top compartment is always destabilised by nutrient cycling. The other compartments are either stabilised if they are at odd distances from the top compartment or destabilised if they are at even distances (see Fig. S2-2 in the supporting information).

\section{Self-regulation}

We explore the effects of self-regulation on food chain stability by considering a nutrient-primary producerherbivore system. In the nutrient-primary producer system presented above, self-regulation directly affects the positive and negative effects of the primary producers on mineral nutrients. This effect leads to a specific response of the system to self-regulation, which is detailed in Fig. S2-7 in the supporting information; thus, we consider more trophic levels to derive more general conclusions on ecosystem functioning. First, the invariability of herbivores $B_{2}$ is constant for most values of self-regulation $D$ but increases and decreases for higher values. The observed increase is due to the decrease in the standard deviation of $B_{2}$ while its biomass remains constant. The final decrease is due 

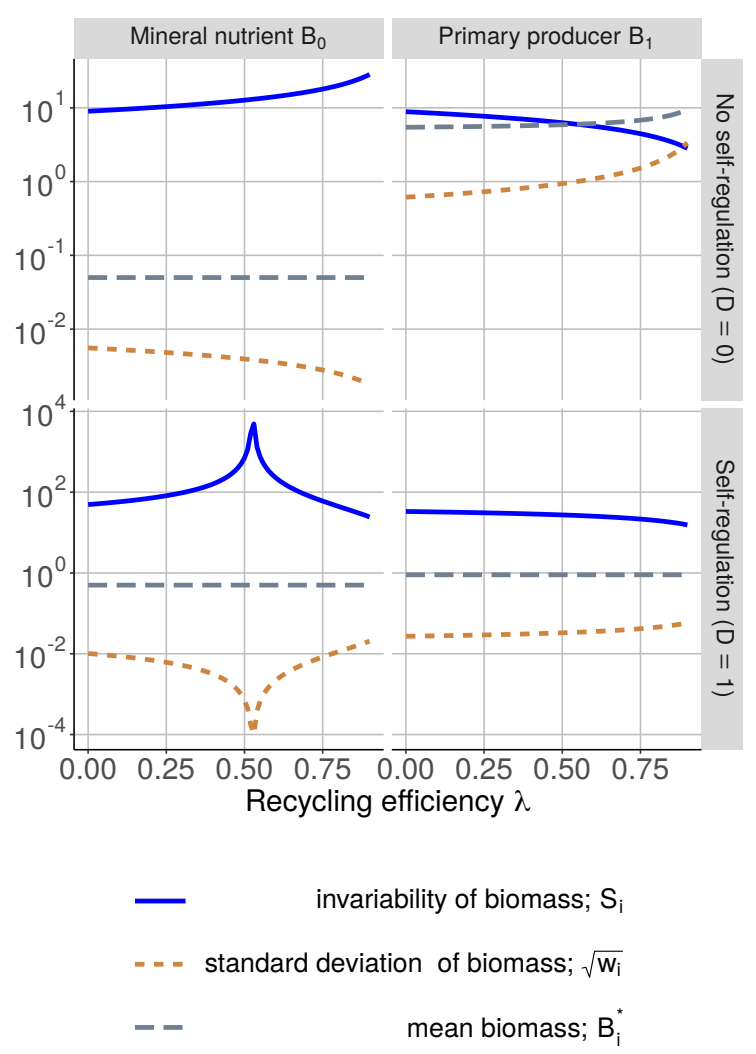

Figure 4: Stability (measured by invariability), standard deviation and biomass of different compartments depending on recycling efficiency $\lambda$ and self-regulation coefficient $D$ in a food chain with a chain length equal to 1 (TL1). Primary producers are perturbed and basal external inputs $I$ are fixed to 0.05 .

to a decrease in herbivore biomass because of intense self-regulation, while the decrease in the standard deviation is less strong (Fig. 6).

The invariability of the mineral nutrients $B_{0}$ increases because of the decrease in the standard deviation, while the nutrient stock remains constant. Again, we can explain this pattern by quantifying the direct and indirect interactions described in Fig. 7. The overall effect of herbivores $B_{2}$ on mineral nutrients $B_{0}$ is mostly positive and the direct recycling effect of $B_{2}$ must prevail as the variations in the standard deviation of the nutrients are similar to those of the herbivores.

Again, we can explain this pattern based on the terms of the Jacobian matrix summarised by Fig. 7A. As there are three compartments, we must consider the direct effect of herbivores on mineral nutrients represented by the recycling of $B_{2}(2)$ and the indirect effect represented by the product of the predation of $B_{2}$ on $B_{1}(1)$ and all the effects of $B 1$ on $B_{0}(3)$ (i.e., recycling of $B_{1}$ and predation of $B_{1}$ on $B_{0}$ ). According to the variations in the terms of the Jacobian matrix, the overall effect of the herbivores $B_{2}$ on the mineral nutrients $B_{0}$ is mostly positive (see Fig. S2-5 in the supporting information). The direct effect of recycling
A
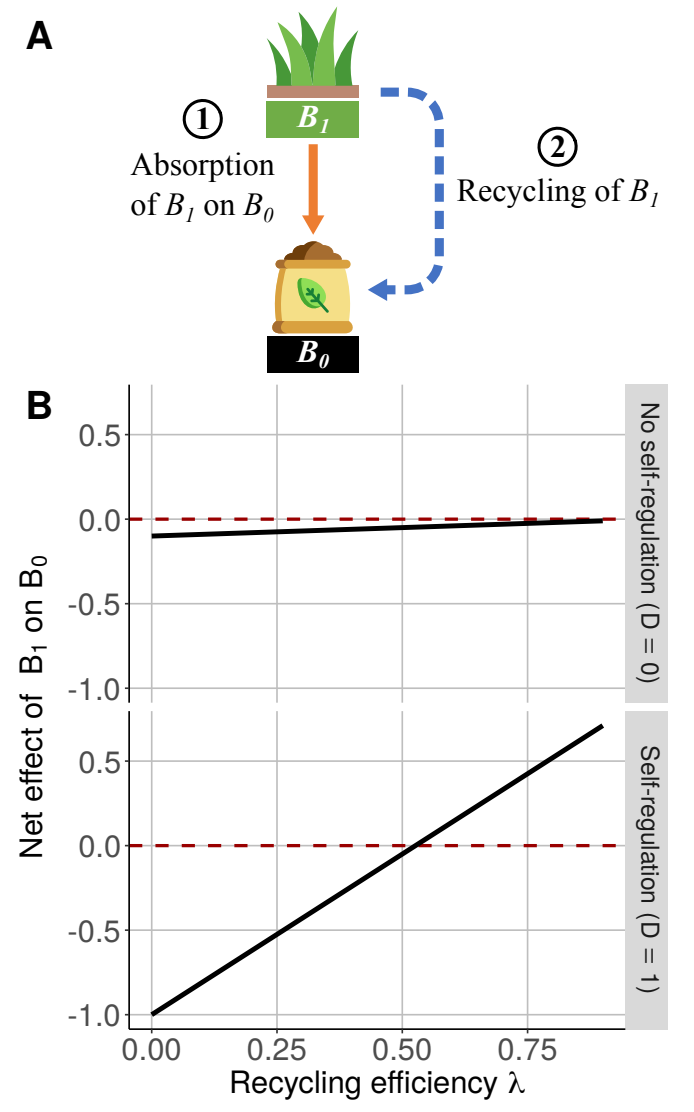

Figure 5: Effects of primary producers on mineral nutrients. A) Schema of the nutrient-primary producer system. The orange arrow represents (1) the negative effect of the absorption of $B_{0}$ by $B_{1}$, and the blue dashed arrow represents (2) the positive recycling effect of $B_{1}$. B) Variations in the direct net effects $((1)+(2))$ of primary producers $B_{1}$ on mineral nutrients $B_{0}$ (equal to element $J_{01}$ of the Jacobian matrix) depending on recycling efficiency $\lambda$ and self-regulation coefficient $D$ in a food chain with a chain length equal to 1 (TL1). The dashed red line represents a null net effect $\left(J_{01}=0\right)$.

by $B_{2}$ must prevail as the variations in the standard deviation of the nutrients are similar to those of the herbivores (Fig. 6).

Similarly, Fig. 7B explains the variations in the invariability of primary producers $B_{1}$. The particular response to self-regulation for $\lambda=1$ is due to the strong indirect effect of herbivore recycling on primary producers coupled with self-regulation differentially affecting $B_{1}$ and $B_{2}$ due to their different abundances (see Fig. S2-5 and the detailed description in the supporting information). For low values of $\lambda$ (i.e., $\lambda<1$ ), recycling efficiency is not high enough to allow perturbations to be transmitted mainly by nutrient cycling. Thus, there is a monotonous increase in the invariability of the primary producers, as self-regulation increases $B_{1}$ by decreasing the top-down control of the primary producers by herbivores. 


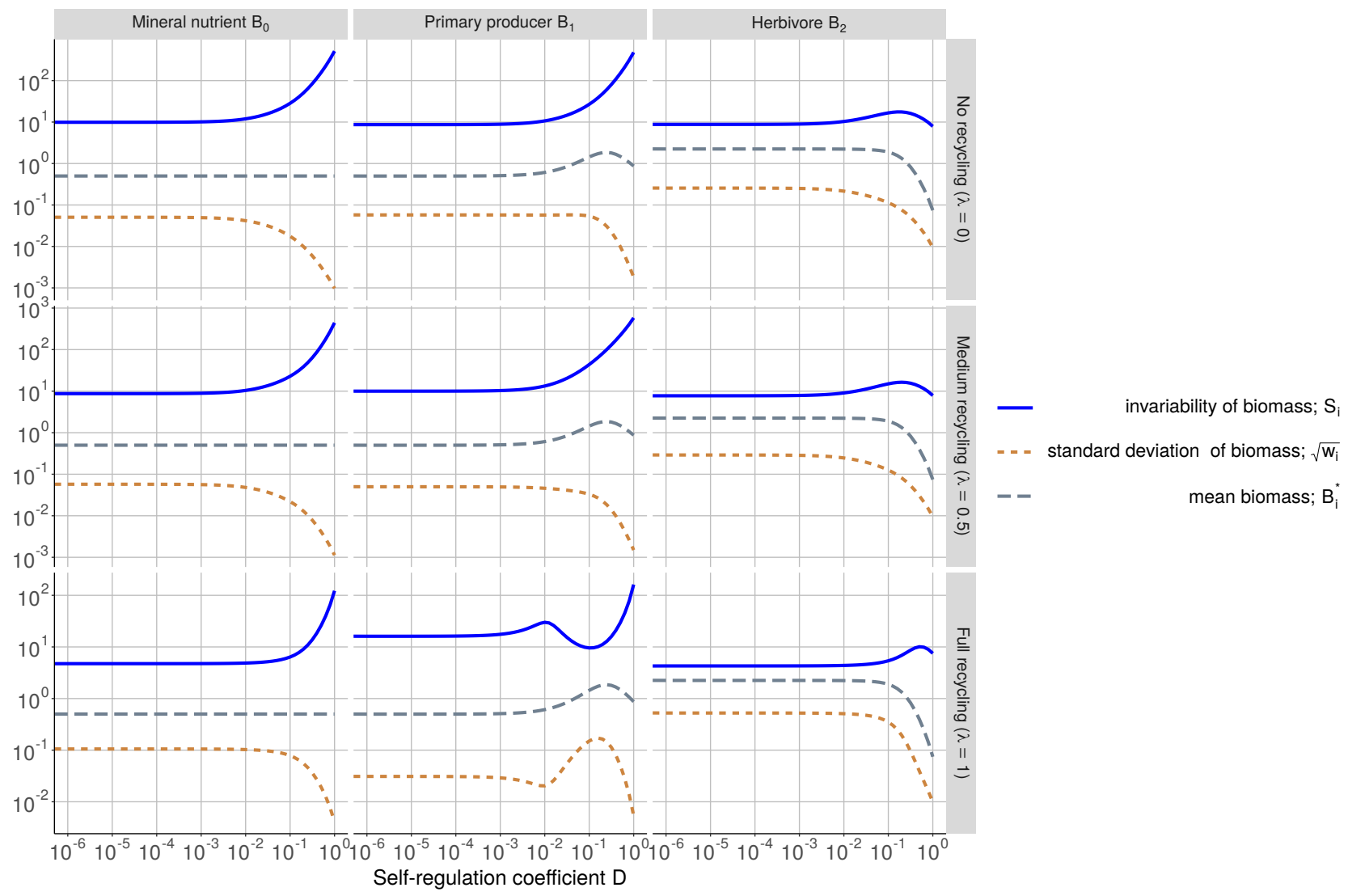

Figure 6: Stability (measured by invariability), standard deviation and biomass of the different compartments depending on recycling efficiency $\lambda$ and self-regulation coefficient $D$ in a food chain with a chain length equal to 2 (TL2). Basal external inputs $I$ are fixed to 0.05 , and predation rate $a_{2}$ is equal to 0.4 when $D=0$ and to 0.401 when $D \neq 0$.

A Effetcs of herbivors $\left(B_{2}\right)$ on mineral nutrients $\left(B_{0}\right)$

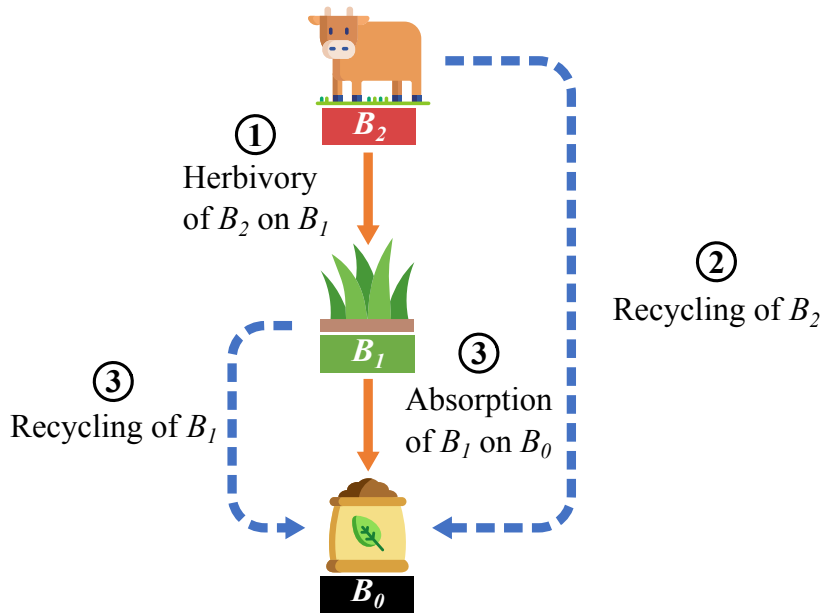

B Effetcs of herbivors $\left(B_{2}\right)$ on primary producers $\left(B_{1}\right)$

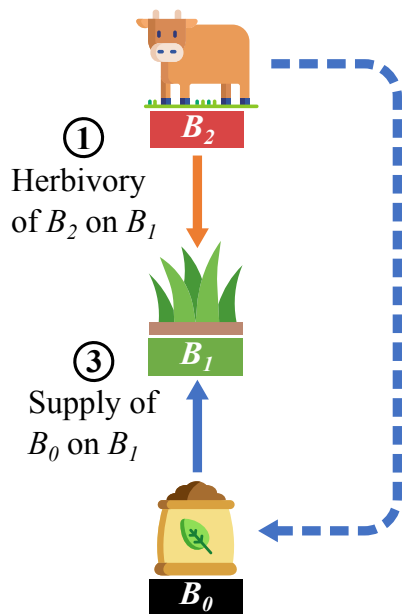

(2)

Recycling of $B_{2}$

Figure 7: Schematic representing the direct and indirect effects of $\mathbf{A}$ ) herbivores on mineral nutrients. Blue arrows represent the positive terms $(2) \lambda\left(m+2 D B_{2}\right)+\lambda\left(1-b_{2}\right) a_{2} B_{1},(3) \lambda\left(m+2 D B_{1}\right)+\lambda\left(1-b_{2}\right) a_{2} B_{2}$. Orange arrows represent the negative terms (1) $-a_{2} B_{1}$ and $(3)-a_{1} B_{0}$. Note that the direct effect of $B_{1}$ on $B_{0}$ (3) is partitioned into its positive and negative terms. B) Effects of herbivores on primary producers. Blue arrows represent the positive terms $(2) \lambda\left(m+2 D B_{2}\right)+\lambda\left(1-b_{2}\right) a_{2} B_{1},(3) a_{1} B_{1}$. Orange arrows represent the negative terms (1) $-a_{2} B_{1}$. Note that the effect of nutrient absorption (3) is now positive because it is considered from the perspective of primary producers. 
A

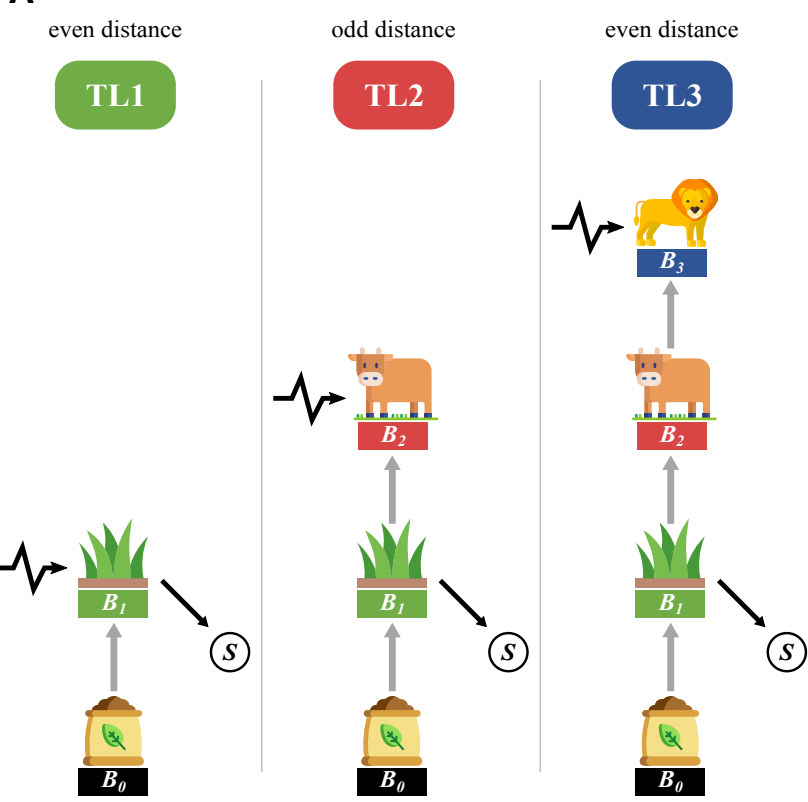

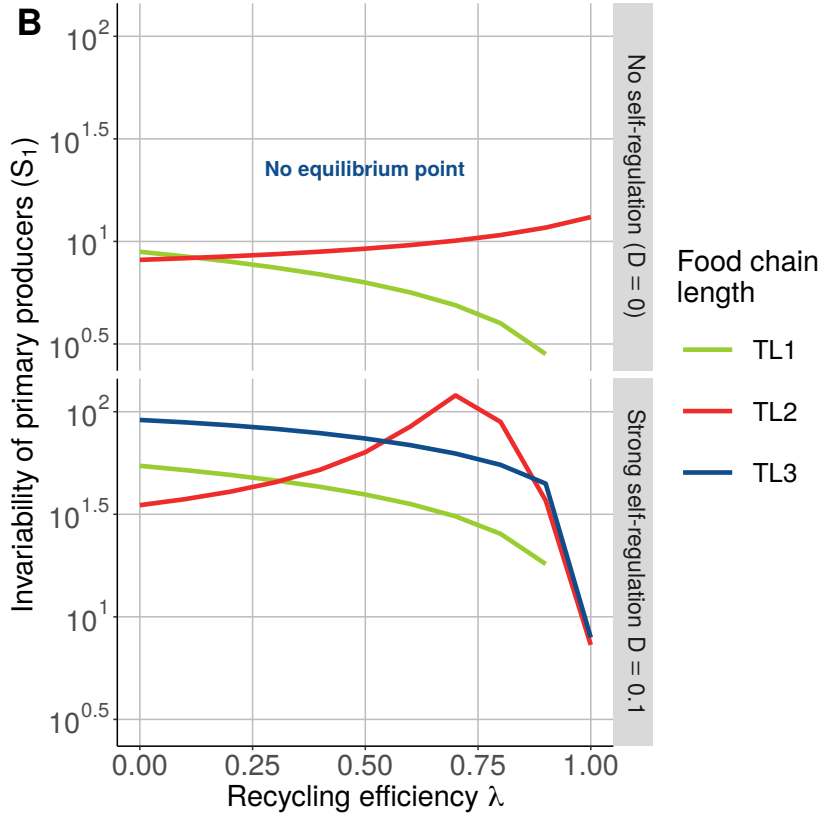

Figure 8: A) Schematic representing the stability $S$ of the primary producers $B_{1}$ depending on chain length which is set to 1,2 or 3 . Black arrows represent perturbations affecting the top species which is at odd (i.e., TL2) or even (i.e., TL1, TL3) distances from primary producers. B) Stability of the primary producers $B_{1}$ depending on recycling efficiency $\lambda$ and self-regulation coefficient $D$ for various chain lengths. Stability is measured by invariability (i.e., inverse of $\mathrm{CV}$ ), which is the ratio between equilibrium biomass $B_{i}^{*}$ and standard deviation $\sigma_{i}$. External nutrient input $I$ is fixed at 0.01 and attack rate $a_{2}$ is equal to 0.401 .

\section{Food chain length}

Food chain length also affects the invariability of each species since we found previously that the response of stability depends on the distance from the perturbed species (which is the highest trophic level in this study, as shown in Fig. 8A). Thus, species at an odd distances from the perturbed specie are stabilised by nutrient cycling because its positive effect mitigates the negative effect of predation (Fig. 8B and Fig. S2-9 in the supporting information). The invariability of primary producers increases with recycling efficiency $\lambda$ when primary producers are at odd distance from the top species (TL2) with a maximum of invariability near $\lambda=0.75$ when self-regulation is strong $(D=0.1) \mathrm{sim}$ ilarly to Fig. 4. In contrast, species at even distances (Tl1 and TL3) from the perturbed species are destabilised by nutrient cycling. In fact, nutrient cycling enhances the transmission of perturbations through a positive bottom-up effect, which adds to the positive effect due to the top-down control of consumers at odd distances (Fig. 7).

\section{Discussion}

Our model aimed to determine the influence of nutrient cycling and self-regulation on food chain stability with a particular focus on feedback effects. First, we showed that nutrient cycling is always destabilising for perturbed species, while lower trophic levels are sta- bilised or destabilised depending on their trophic distance from the perturbed species. Thus, nutrient cycling is stabilising for species at odd distances whereas it is destabilising for species at even distances. Second, we showed that self-regulation modulates the effects of nutrient cycling by decreasing top-down control and increasing flows of recycled nutrients.

\section{Recycling efficiency}

Our main result is that nutrient cycling has a stabilising or a destabilising effect on each species depending on its trophic distances from the perturbed species. More specifically, species at odd distances are stabilised while species at even distances are destabilised by nutrient cycling (Fig. 8). The key mechanisms involved are, on the one hand, the top-down control of consumers on resources, which leads to trophic cascades (Hairston et al., 1960; Fretwell and Barach, 1977; Oksanen et al., 1981), and, on the other hand, the positive bottom-up effect of recycled nutrients (Leroux and Loreau, 2010; Loreau, 2010). In fact, nutrient cycling dampens the negative effects cascading on species at odd distances from the top predator by adding a positive bottom-up effect of predators on their prey (Figs. 4 and 5). This mechanism smooths the distribution of biomass CV predicted by Shanafelt and Loreau (2018), who found a strong difference between controlled and uncontrolled species and demonstrates that ecosystem processes can deeply impact community dynam- 
ics. Conversely, nutrient cycling destabilises species at even distances as the positive bottom-up effects add to the positive cascading top-down effects, thus enhancing the transmission of perturbations. This interplay between nutrient cycling and cascading effects has also been observed for biomass by Leroux and Loreau (2010), who found that nutrient cycling increases the biomass of species at even distances from the top consumer and not that of species at even distances (controlled species).

However, these results are not consistent with the results of Quévreux et al. (2021b), who did not find this pattern. This discrepancy is due to the presence of limit cycles in their system whereas we consider stochastic perturbations in the vicinity of equilibrium. In systems with limit cycles, nutrient cycling creates new couplings of the phase of each predator-prey oscillator, thus altering the amplitude of oscillations and biomass CVs. On the other hand, stochastic perturbations ripple across the food chain and lead to trophic cascade-like patterns of stability. This shows that the effect of nutrient cycling on ecosystem dynamics depends on the state of the system and future studies should consider this aspect in their results. In addition, we show that asymptotic resilience is not representative of the response of the entire ecosystem (see Figs. S2-1, S2-4, S2-6 and S2-8 in the supporting information), but is mainly driven by a single compartment (Arnoldi et al., 2016; Haegeman et al., 2016). Considering invariability as a result of stochastic perturbations gives a detailed description of the stability of each ecosystem compartment.

Our results demonstrate the importance of indirect effects since the indirect positive effect of nutrient cycling on resources can overcome the direct negative effect of consumers (Fig. 5). The key role of long and weak loops in the stability of interaction networks has been already identified (McCann et al., 1998; Neutel et al., 2002) but Neutel and Thorne (2014) showed that feedback loops generated by nutrient cycling generally have a neutral effect on asymptotic resilience. This discrepancy can be explained by two factors. First, Neutel and Thorne (2014) did not consider self-regulation when calculating asymptotic resilience, while we show that its absence strongly reduces the response of asymptotic resilience to nutrient cycling (see Figs. S2-1 and S2-4 in the supporting information). Second, the complexity of food webs they analysed may blur the feedback effects of nutrient cycling. For instance, omnivory, by increasing the number of direct and indirect interactions, may weaken the net outcome of nutrient cycling. Such an effect can be observed with food chain length since the maximum of invariability observed for TL1 in Fig. 4 is less clear for TL2 in Fig. S2-2. Ultimately, nutrient cycling has almost no effect on stability in food webs with numerous interacting species (Quévreux et al., 2021b).

Finally, our model represents nutrient cycling in a very simple ways in order to grasp the fundamental effects of feedback loops generated by nutrient cycling on food web stability. Actually, nutrient cycling is much more complex since nutrients are also excreted as detritus that are degraded by decomposers (Moore et al., 2004). Decomposers and their consumers form the brown food web, which interacts with the green food web that relies on photosynthetic primary producers, through carbon and nutrient availability for instance (Daufresne and Loreau, 2001; Daufresne et al., 2008; Joint et al., 2002; Danger et al., 2007; Cherif and Loreau, 2013). These interactions strongly affect ecosystem functioning (Attayde and Ripa, 2008; Zou et al., 2016; Buchkowski et al., 2019) and food web stability (Gounand et al., 2014; Mougi, 2020). However, Mougi (2020) considered asymptotic resilience to measure stability and future studies should study the response to stochastic perturbations to fully understand the effects of nutrient cycling on ecosystem stability.

\section{Self-regulation}

First, we found that self-regulation is generally stabilising when it is present for two or more trophic levels (Fig. 6), although it becomes destabilising and can lead to species extinction if it is too strong (Barabás et al., 2017). The stabilising effect of self-regulation is due to a decrease in the ratio of interspecific to intraspecific interactions which leads to a lesser transmission of perturbations. However, the effects of self-regulation seem heavily dependent on food chain lengths (Fig. 8). In a simple mineral nutrient-primary producer system, self-regulation has no effect on stability, as equilibrium biomass and standard deviation vary with $D$ at the same rate (see Fig. S2-7 in the supporting information). For the system with three trophic levels, the effect of self-regulation is unclear because at a low coefficient of self-regulation $D$, the system does not reach equilibrium (see Fig. S2-9 in the supporting information).

In our model, self-regulation strongly interacts with nutrient cycling as it increases the quantity of nutrients released by organisms. In fact, the balance between the two mechanisms described earlier depends on the intensity of the self-regulation. For instance, nutrient cycling tends to reduce the stabilising effect of self-regulation on perturbed species and trophic levels at even distances from the perturbed species by making the perturbation loop inside the ecosystem. To our knowledge, most of the existing models studying nutrient cycling do not consider self-regulation (DeAngelis et al., 1989; Nakajima and DeAngelis, 1989; Loreau, 1994; Leroux and Loreau, 2010; McCann, 2012 but see Quévreux et al., 2021b), but given its interactions with nutrient cycling and its central role in the overall dynamics of food chains (Barbier and Loreau, 2019), self-regulation should be considered in future models combining community ecology and ecosystem functioning.

Finally, self-regulation summarises various mecha- 
nisms and is considered as an additional mortality term in our model. However, it can be seen as a decrease in the resource uptake rate, as in the Beddington-DeAngelis functional response (Beddington, 1975; DeAngelis et al., 1975), which considers mutual interference of predators. In future studies using such a model, we would expect self-regulation to decrease the quantity of recycled nutrients by reducing material flows between species, which would decrease the feedback effect of nutrient cycling. In addition, the Beddington-DeAngelis functional response alters interspecific interactions since it is nonlinear, while our study is based a linear functional response. However, we should only expect minor changes from this point for two main reasons. First, type II functional responses do not alter drastically biomass distribution among species at equilibrium (Barbier and Loreau, 2019). Second, since we consider small perturbations in the vicinity of equilibrium, our system is linearised and nonlinear functional responses act as linear Lotka-Volterra predator-prey interactions in the vicinity of the equilibrium.

\section{Empirical testing}

Testing our results empirically is challenging because nutrient cycling is a fundamental ecosystem process that is not easy to measure and control. Nevertheless, microcosm or mesocosm experiments offer promising opportunities. For instance, Harrault et al. (2014) performed an aquatic mesocosm experiment in which they added sediments collected in a previous experiment to test the bottom-up effects of the quality of dead organic matter on pelagic food webs. In this spirit, removing the sediments produced in a mesocosm is equivalent to reducing recycling efficiency $\lambda$ in our model and adding external sediments is equivalent to the compensation explained in Fig. 3. However, in future experiments, recycling efficiency $\lambda$ should not be considered equal among trophic levels. Harrault et al. (2012) demonstrated that detritus from mesocosms with fish were more degradable than detritus from mesocosms without fish. This increased degradability is equivalent to a higher $\lambda$ and led to a stronger positive effect of nutrient cycling on phytoplankton (Harrault et al., 2014), as observed by previous experiments (Vanni et al., 1997; Vanni and Layne, 1997; Attayde and Hansson, 2001).

The crossed effects of nutrient cycling and selfregulation could be tested in microcosm experiments because microorganisms can be easily killed and turned into labile organic matter by microwaving a sample from microcosms (Harvey et al., 2016, 2020; Jacquet et al., 2020; Jacquet and Altermatt, 2020). Thus, controlling the size of the microwaved sample depending on the density of protists would mimic variations in self-regulation, and reinjecting or not the sample would link self-regulation to nutrient cycling.

\section{Conclusion}

Our study shows that the effects of nutrient cycling on ecosystem stability depend on the measure of stability since our results diverge from those in the existing literature using different measures. By considering stochastic perturbations, we found that nutrient cycling can be stabilising or destabilising depending on the positive and negative direct or indirect effects of the perturbed species on each species. This results in a trophic cascade pattern since species at even trophic distances from the perturbed species are destabilised, while species at odd distances are stabilised.

We also show that self-regulation, which is usually considered in a pure community ecology context, strongly interacts with nutrient cycling. By increasing the quantity of recycled nutrients and reducing the strength of top-down control, self-regulation strengthens the positive feedback loops generated by nutrient cycling. Thus, considering self-regulation should strongly impact the results of future studies considering similar models.

Our results provide broader insight into the mechanisms governing ecosystem stability and open models considering a more realistic representation of nutrient cycling, such as with detritus and decomposers.

\section{Data accessibility}

The Python code of the simulations and $\mathrm{R}$ code of the figures are available on GitHub.

\section{Acknowledgement}

This work was supported by the TULIP Laboratory of Excellence (ANR-10-LABX-41) and by the BIOSTASES Advanced Grant, funded by the European Research Council under the European Union's Horizon 2020 research and innovation programme (666971). We also thank Matthieu Barbier for his useful advises and comments and Shawn Leroux and the anonymous reviewer for their suggestions to improve this manuscript.

\section{Authors' contributions}

Conceptualisation (KT, PQ, ML) - Funding acquisition (ML) - Model analysis (KT, PQ, ML) - Coding simulation (KT) - Supervision (PQ, ML) - Original draft writing (KT, PQ) - Review \& editing (PQ, ML)

\section{References}

Arnold, L. (1974). Stochastic differential equations: Theory and applications. New York: Wiley. 
Arnoldi, J., Bideault, A., Loreau, M., \& Haegeman, B. (2018). How ecosystems recover from pulse perturbations: A theory of short- to long-term responses. Journal of Theoretical Biology, 436, 79-92. doi:10.1016/j.jtbi.2017.10.003

Arnoldi, J., Loreau, M., \& Haegeman, B. (2016). Resilience, reactivity and variability: A mathematical comparison of ecological stability measures. Journal of Theoretical Biology, 389, 4759. doi:10.1016/j.jtbi.2015.10.012

Arnoldi, J., Loreau, M., \& Haegeman, B. (2019). The inherent multidimensionality of temporal variability: How common and rare species shape stability patterns. Ecology Letters, 22(10), 15571567. doi: $10.1111 /$ ele. 13345

Attayde, J. L., \& Hansson, L.-A. (2001). Fish-mediated nutrient recycling and the trophic cascade in lakes. Canadian Journal of Fisheries and Aquatic Sciences, 58(10), 1924-1931. doi:10.1139 / f01128

Attayde, J. L., \& Ripa, J. (2008). The coupling between grazing and detritus food chains and the strength of trophic cascades across a gradient of nutrient enrichment. Ecosystems, 11(6), 980 990. doi:10.1007/s10021-008-9174-8

Barabás, G., Michalska-Smith, M. J., \& Allesina, S. (2017). Self-regulation and the stability of large ecological networks. Nature Ecology $\&$ Evolution, 1(12), 1870-1875. Number: 12 Publisher: Nature Publishing Group. doi:10.1038/s41559-0170357-6

Barbier, M., \& Loreau, M. (2019). Pyramids and cascades: A synthesis of food chain functioning and stability. Ecology Letters, 22(2), 405-419. doi:10. 1111/ele.13196

Beddington, J. R. (1975). Mutual interference between parasites or predators and its effect on searching efficiency. The Journal of Animal Ecology, 44(1), 331. doi:10.2307/3866

Begon, M., Harper, J. L., \& Townsend, C. R. (1986a). Ecology: Individuals, populations and communities. OCLC: 833046410. Oxford: Blackwell.

Begon, M., Mortimer, M., \& Thompson, D. J. (1986b). Population Ecology a unified study of animals and plants. OCLC: 1185818106. Retrieved January 8, 2021, from https://nbn-resolving.org/ urn:nbn:de:101:1-201501027681

Brose, U., Williams, R. J., \& Martinez, N. D. (2006). Allometric scaling enhances stability in complex food webs. Ecology Letters, 9(11), 1228-1236. doi:10.1111/j.1461-0248.2006.00978.x

Brown, D. H., Ferris, H., Plant, R., \& Fu, S. (2004). Modeling direct positive feedback between predators and prey. Theoretical Population Biology, 65(2), 143-152. doi:10.1016/j.tpb. 2003.09.004

Buchkowski, R. W., Leroux, S. J., \& Schmitz, O. J. (2019). Microbial and animal nutrient limitation change the distribution of nitrogen within coupled green and brown food chains. Ecology, 100(5), e02674. _eprint: https://esajournals.onlinelibrary.wiley.com/doi/pdf/10.1002/e doi:10.1002/ecy. 2674

Cherif, M., \& Loreau, M. (2013). Plant-herbivore-decomposer stoichiometric mismatches and nutrient cycling in ecosystems. Proc. R. Soc. B, $280(1754), 20122453$. doi:10.1098/rspb.2012.2453

Danger, M., Leflaive, J., Oumarou, C., Ten-Hage, L., \& Lacroix, G. (2007). Control of phytoplanktonbacteria interactions by stoichiometric constraints. Oikos, 116(7), 1079-1086. doi:10.1111/ j.0030-1299.2007.15424.x

Daufresne, T., Lacroix, G., Benhaim, D., \& Loreau, M. (2008). Coexistence of algae and bacteria: A test of the carbon hypothesis. Aquatic Microbial Ecology, 53, 323-332. doi:10.3354/ame01254

Daufresne, T., \& Loreau, M. (2001). Ecological stoechiometry, primary producer-decomposer interactions, and ecosystem persistence. Ecology, 82(11), 3069-3082. doi:10.1890/0012-9658(2001) 082[3069:ESPPDI]2.0.CO;2

DeAngelis, D. L. (1980). Energy flow, nutrient cycling, and ecosystem resilience. Ecology, 61(4), 764. doi: $10.2307 / 1936746$

DeAngelis, D. L. (1992). Dynamics of nutrient cycling and food webs (1. ed). Population and community biology series. London: Chapman \& Hall.

DeAngelis, D. L., Goldstein, R. A., \& O'Neill, R. V. (1975). A model for tropic interaction. Ecology, 56(4), 881. doi: $10.2307 / 1936298$

DeAngelis, D. L., Mulholland, P. J., Palumbo, A. V., Steinman, A. D., Huston, M. A., \& Elwood, J. W. (1989). Nutrient dynamics and food-web stability. Annual Review of Ecology and Systematics, 20(1), 71-95. doi:10.1146/annurev.es.20. 110189.000443

Fretwell, S. D., \& Barach, A. L. (1977). The regulation of plant communities by the food chains exploiting them. Perspectives in Biology and Medicine, 20 (2), 169-185. doi:10.1353/pbm.1977.0087

Gounand, I., Mouquet, N., Canard, E., Guichard, F., Hauzy, C., \& Gravel, D. (2014). The paradox of enrichment in metaecosystems. The American Naturalist, 184 (6), 752-763. doi:10.1086/678406

Grimm, V., \& Wissel, C. (1997). Babel, or the ecological stability discussions: An inventory and analysis of terminology and a guide for avoiding confusion. Oecologia, 109(3), 323-334. doi:10.1007/ s004420050090

Gross, K., Cardinale, B. J., Fox, J. W., Gonzalez, A., Loreau, M., Wayne Polley, H., ... van Ruijven, J. (2014). Species richness and the temporal stability of biomass production: A new analysis of recent biodiversity experiments. The American Naturalist, 183(1), 1-12. doi:10.1086/673915

Haegeman, B., Arnoldi, J.-F., Wang, S., de Mazancourt, C., Montoya, J. M., \& Loreau, M. (2016). 
Resilience, invariability, and ecological stability across levels of organization. bioRxiv. doi:10 . $1101 / 085852$

Hairston, N. G., Smith, F. E., \& Slobodkin, L. B. (1960). Community structure, population control, and competition. The American Naturalist, 94(879), 421-425. doi:10.1086/282146

Harrault, L., Allard, B., Danger, M., MaunouryDanger, F., Guilpart, A., \& Lacroix, G. (2012). Influence of food-web structure on the biodegradability of lake sediment. Freshwater Biology, 57(11), 2390-2400. doi:10.1111/fwb.12020

Harrault, L., Allard, B., Mériguet, J., Carmignac, D., Huon, S., Gauzens, B., \& Lacroix, G. (2014). Bottom-up effects of lake sediment on pelagic food-web compartments: A mesocosm study. Freshwater Biology, 59(8), 1695-1709. doi:10 . 1111/fwb. 12375

Harvey, E., Gounand, I., Fronhofer, E. A., \& Altermatt, F. (2020). Metaecosystem dynamics drive community composition in experimental, multilayered spatial networks. Oikos, 129(3), 402-412. doi:10.1111/oik.07037

Harvey, E., Gounand, I., Ganesanandamoorthy, P., \& Altermatt, F. (2016). Spatially cascading effect of perturbations in experimental metaecosystems. Proceedings of the Royal Society B: Biological Sciences, 283(1838), 20161496. doi:10. 1098/rspb.2016.1496

Heckmann, L., Drossel, B., Brose, U., \& Guill, C. (2012). Interactive effects of body-size structure and adaptive foraging on food-web stability: Body size, adaptivity and food-web stability. Ecology Letters, 15(3), 243-250. doi:10.1111/j. 1461-0248.2011.01733.x

Jacquet, C., \& Altermatt, F. (2020). The ghost of disturbance past: Long-term effects of pulse disturbances on community biomass and composition. Proceedings of the Royal Society B: Biological Sciences, 287(1930), 20200678. doi:10.1098/ rspb.2020.0678

Jacquet, C., Gounand, I., \& Altermatt, F. (2020). How pulse disturbances shape size-abundance pyramids. Ecology Letters, 23(6), 1014-1023. doi:10. 1111/ele.13508

Joint, I., Henriksen, P., Fonnes, G., Bourne, D., Thingstad, T., \& Riemann, B. (2002). Competition for inorganic nutrients between phytoplankton and bacterioplankton in nutrient manipulated mesocosms. Aquatic Microbial Ecology, 29, 145-159. doi:10.3354/ame029145

Leroux, S. J., \& Loreau, M. (2010). Consumermediated recycling and cascading trophic interactions. Ecology, 91 (7), 2162-2171.

Loreau, M. (1998). Ecosystem development explained by competition within and between material cycles. Proceedings of the Royal Society B: Biological Sciences, 265 (1390), 33-38. doi:10.1098/ rspb. 1998.0260
Loreau, M. (1994). Material cycling and the stability of ecosystems. The American Naturalist, 143(3), 508-513.

Loreau, M. (1995). Consumers as maximizers of matter and energy flow in ecosystems. The American Naturalist, 145(1), 22-42. doi:10.1086/285726

Loreau, M. (2010). Material cycling and the overall functioning of ecosystems. In From populations to ecosystems: Theoretical foundations for a new ecological synthesis (Vol. 46, 46, pp. 164-195). Monographs in population biology. doi:10.2307/ j.ctt7s78j.10

McCann, K. S. (2000). The diversity-stability debate. Nature, 405(6783), 228-233. doi:10.1038/ 35012234

McCann, K. S. (2012). Adding the ecosystem. In Food Webs (pp. 189-200). doi:10.2307/j.ctt7rr0s.14

McCann, K. S., Hastings, A., \& Huxel, G. R. (1998). Weak trophic interactions and the balance of nature. Nature, 395(6704), 794-798. doi:10.1038/ 27427

Moore, J. C., Berlow, E. L., Coleman, D. C., Ruiter, P. C., Dong, Q., Hastings, A., ... Wall, D. H. (2004). Detritus, trophic dynamics and biodiversity. Ecology Letters, 7(7), 584-600. doi:10.1111/ j.1461-0248.2004.00606.x

Moore, J. C., \& Ruiter, P. C. d. (2012). Energetic Food Webs: An analysis of real and model ecosystems. OUP Oxford.

Mougi, A. (2020). Coupling of green and brown food webs and ecosystem stability. Ecology and Evolution, ece3.6586. doi:10.1002/ece3.6586

Nakajima, H., \& DeAngelis, D. L. (1989). Resilience and local stability in a nutrient-limited resourceconsumer system. Bulletin of Mathematical Biology, 51(4), 501-510. doi:10.1007/BF02460087

Neutel, A.-M., Heesterbeek, J. A. P., \& De Ruiter, P. C. (2002). Stability in real food webs: Weak links in long loops. Science, 296(5570), 11201123. doi:10.1126/science. 1068326

Neutel, A.-M., \& Thorne, M. A. (2014). Interaction strengths in balanced carbon cycles and the absence of a relation between ecosystem complexity and stability. Ecology Letters, 17(6), 651-661. doi:10.1111/ele.12266

O'Neill, R. V. (1976). Ecosystem persistence and heterotrophic regulation. Ecology, 57(6), 1244. doi:10.2307/1935048

Oksanen, L., Fretwell, S. D., Arruda, J., \& Niemela, P. (1981). Exploitation ecosystems in gradients of primary productivity. The American Naturalist, 118(2), 240-261. doi:10.1086/283817

Picoche, C., \& Barraquand, F. (2019). How selfregulation, the storage effect, and their interaction contribute to coexistence in stochastic and seasonal environments. Theoretical Ecology, 12(4), 489-500. doi:10.1007/s12080-019-0420-9

Picoche, C., \& Barraquand, F. (2020). Strong selfregulation and widespread facilitative interac- 
tions in phytoplankton communities. Journal of Ecology, 1365-2745.13410. doi:10.1111/13652745.13410

Quévreux, P., Barbier, M., \& Loreau, M. (2021a). Synchrony and perturbation transmission in trophic metacommunities. The American Naturalist, 714131. doi:10.1086/714131

Quévreux, P., Barot, S., \& Thébault, É. (2021b). Interplay between the paradox of enrichment and nutrient cycling in food webs. Oikos, 130(1), 95109. doi:10.1111/oik.07937

Rakowski, C. J., Farrior, C. E., Manning, S. R., \& Leibold, M. A. (2019). Predator complementarity dampens variability of phytoplankton biomass in a diversity-stability trophic cascade. bioRxiv. doi:10.1101/851642

Shanafelt, D. W., \& Loreau, M. (2018). Stability trophic cascades in food chains. Royal Society Open Science, 5(11), 180995. doi:10.1098/rsos. 180995

Sterner, R. W., Bajpai, A., \& Adams, T. (1997). The enigma of food chain length: Absence of theoretical evidence for dynamic constraints. Ecology, 78(7), 2258-2262. Publisher: Ecological Society of America. doi:10.2307/2265962

Tilman, D., Reich, P. B., \& Knops, J. M. H. (2006). Biodiversity and ecosystem stability in a decadelong grassland experiment. Nature, 441(7093), 629-632. doi:10.1038/nature04742

Ulanowicz, R. E. (1990). Aristotelean causalities in ecosystem development. Oikos, $57(1)$, 42. doi:10. $2307 / 3565734$

Vanni, M. J. (2002). Nutrient cycling by animals in freshwater ecosystems. Annual Review of Ecology and Systematics, 33, 341-370. Retrieved May 11, 2016, from http://www.jstor.org/stable/3069266

Vanni, M. J., \& Layne, C. D. (1997). Nutrient recycling and herbivory as mechanisms in the "top-down" effect of fish on algae in lakes. Ecology, 78(1), 21-40. doi:10 . 1890 / 0012-9658(1997 ) 078[0021 : NRAHAM]2.0.CO;2

Vanni, M. J., Layne, C. D., \& Arnott, S. E. (1997). "Top-down" trophic interactions in lakes: Effects of fish on nutrient dynamics. Ecology, 78(1), 1-20. doi:10 . 1890 / 0012 - 9658(1997 ) 078[0001 : TDTIIL]2.0.CO;2

Veldhuis, M. P., Berg, M. P., Loreau, M., \& Olff, H. (2018a). Ecological autocatalysis: A central principle in ecosystem organization? Ecological Monographs, 88(3), 304-319. doi:10.1002/ ecm. 1292

Veldhuis, M. P., Gommers, M. I., Olff, H., \& Berg, M. P. (2018b). Spatial redistribution of nutrients by large herbivores and dung beetles in a savanna ecosystem. Journal of Ecology, 106(1), 422-433. _eprint: https://besjournals.onlinelibrary.wiley.com/doi/pdf/10.1111/13652745.12874. doi:10.1111/1365-2745.12874
Wang, S., Haegeman, B., \& Loreau, M. (2015). Dispersal and metapopulation stability. PeerJ, 3, e1295. doi:10.7717/peerj. 1295

Yodzis, P. (1981). The stability of real ecosystems. $N a$ ture, 289(5799), 674-676. doi:10.1038/289674a0

Zou, K., Thébault, É., Lacroix, G., \& Barot, S. (2016). Interactions between the green and brown food web determine ecosystem functioning. Functional Ecology, 30(8), 1454-1465. doi:10.1111/ 1365-2435.12626 


\title{
Nutrient cycling and self-regulation determine food web stability
}

\author{
Kevin Theis ${ }^{1, *}$, Pierre Quévreux ${ }^{1, *}$, and Michel Loreau ${ }^{1}$ \\ ${ }^{1}$ Theoretical and Experimental Ecology Station, UPR 2001, CNRS, 09200 Moulis, France \\ * authors contributed equally
}

\section{S1 Complementary material and methods}

\section{S1-1 Calculation of the covariance matrix}

$C^{*}$ is calculated thanks to a Kronecker products method (Nip et al., 2013). Kronecker products of the $A$ matrix of dimensions $m * n$ with the $B$ matrix of dimensions $p * q$, denoted $A \otimes B$, is the matrix of dimensions $m p * n q$ such as:

$A \otimes B=\left(\begin{array}{ccc}a_{11} B & \ldots & a_{1 n} B \\ \vdots & \ddots & \vdots \\ a_{m 1} & \ldots & a_{m n} B\end{array}\right)$

We define $\left(C^{*}\right)_{s}$ and $\left(T V_{E} T^{t}\right)_{s}$ as column vectors of $C^{*}$ and $T V_{E} T^{t}$ respectively. Equation (4) can be solved.

$$
\begin{aligned}
& (J \otimes I+I \otimes J) C_{s}^{*}=-\left(T V_{E} T^{t}\right)_{s} \\
& C_{s}^{*}=-(J \otimes I+I \otimes J)^{-1}\left(T V_{E} T^{t}\right)_{s}
\end{aligned}
$$

\section{S1-2 Nutrient availability and recycling importance}

We parametrise our model so that the quantity of recycled $I_{\text {recymax }}$ is larger than external inputs $I$. Equations (8) and (9) are the analytic expressions of the ratio of these two quantities in a food chain with mineral nutrients and primary producers only (TL1) and a food chain with mineral nutrients, primary producers and herbivores (TL2) respectively.

$$
\begin{aligned}
& \frac{I_{\text {recymax }}}{I}=\frac{a_{1}\left(I a_{1}-m \ell\right)}{D \ell^{2}} \\
& \frac{I_{\text {recymax }}}{I}=\frac{a_{1}\left(a_{1} D I+a_{2} \ell m-D \ell m\right)}{\left(a_{2}^{2} b_{2}+D^{2}\right) \ell^{2}}
\end{aligned}
$$

Nutrient uptake by primary producer $a_{1}$ is a major factor controlling this ratio and nutrient cycling can have a significant impact on ecosystem dynamics only if primary producers take up nutrients efficiently.

\section{S1-3 Asymptotic resilience}

Asymptotic resilience is calculated as the opposite of the real part of the lead eigen value of the Jacobian matrix of the system at equilibrium. It represents the asymptotic return rater to equilibrium after a small perturbation, i.e. the return rate after a long time. In addition, the elements of the lead eigen vector (i.e. the

corresponding author: pierre.quevreux@cri-paris.org 
eigen vector associated with the lead eigen value) represent the contribution of each species to this asymptotic rate. Here an example for a system with two interacting compartments:

$V_{\text {lead }}=\left(\begin{array}{l}v_{1} \\ v_{2}\end{array}\right) \quad \begin{aligned} & v_{1}: \text { contribution of species } 1 \\ & v_{2}: \text { contribution of species } 2\end{aligned}$

Thus, if $v_{1} \gg v_{2}$, species 1 drives asymptotic resilience in the system and the returns rate to equilibrium of the systems reflects the returns rate of species 1 (see Arnoldi et al. (2016) for more details).

In the following, we will consider the relative contribution $v_{r, i}$ of each of the $n$ compartments of the ecosystem to the lead eigen vector, with $\left\|\Re\left(v_{i}\right)\right\|$ the absolute value of the real part of element $v_{i}$ of the lead eigen vector:

$$
v_{r, i}=\frac{\left\|\Re\left(v_{i}\right)\right\|}{\sum_{j=1}^{n}\left\|\Re\left(v_{j}\right)\right\|}
$$

\section{References}

Arnoldi, J., Loreau, M., \& Haegeman, B. (2016). Resilience, reactivity and variability: A mathematical comparison of ecological stability measures. Journal of Theoretical Biology, 389, 47-59. doi:10.1016/j.jtbi. 2015.10.012

Nip, M., Hespanha, J. P., \& Khammash, M. (2013). Direct numerical solution of algebraic Lyapunov equations for large-scale systems using Quantized Tensor Trains. In 52nd IEEE Conference on Decision and Control (pp. 1950-1957). doi:10.1109/CDC.2013.6760167 


\section{S2 Complementary results}

\section{S2-1 Recycling efficiency}

\section{Jacobian matrix}

The variations in Fig. 4 can be explained analytically by element $J_{01}$ of the Jacobian matrix since it represents how changes in primary producer biomass $B_{1}$, which is the perturbed specie, influence mineral nutrient $B_{0}$ dynamics.

$J_{01}=\underbrace{-a_{1} B_{0}}_{\text {absorption effect }(1)}+\underbrace{\lambda\left(m+2 D B_{1}\right)}_{\text {recycling effect (2) }}$

When $J_{01}$ is negative, the negative effect of absorption overwhelms the positive effect of recycling and perturbations are mainly transmitted from $B_{1}$ to $B_{0}$ through absorption. As recycling efficiency $\lambda$ increases, the recycling effect becomes stronger until $J_{01}=0$, which corresponds to the situation where the recycling effect totally offsets the absorption effect (Fig. 5B in the main text). Finally, the value of $J_{01}$ becomes positive for high values of $\lambda$ and the perturbation is mainly transmitted by nutrient cycling.

\section{Asymptotic resilience}

Asymptotic resilience is commonly used to assess ecosystem stability (McCann, 2000), but recent studies have revealed that it can be heavily biased by particular elements of ecosystems (Arnoldi et al., 2016; Haegeman et al., 2016). The calculation of asymptotic resilience and the associated variables is detailed in section S1-3 in the supporting information.

In a food chain with one trophic level (nutrients $B_{0}$ and primary producers $B_{1}$ ), when there is no self-regulation $(D=0)$, resilience slightly decreases with recycling efficiency $\lambda$ (Fig. S2-1A). This decrease is similar to the variations of the invariability of primary producers (Fig. 4) because primary producers $\left(B_{1}\right)$ contribute for nearly $100 \%$ to the lead eigen vector (Fig. S2-1B), which means that asymptotic resilience is driven by primary producers.

When self-regulation is strong $(D=1)$, asymptotic resilience is high compared to the situation without selfregulation and is first independent of recycling efficiency $\lambda$ (Fig. S2-1A). For $\lambda$ close to 0.5, asymptotic resilience sharply decreases with recycling efficiency $\lambda$. This sharp decreases is paralleled by a transitory increase in the contribution of primary producers $\left(B_{1}\right)$ and a decrease in the contribution of mineral nutrients $\left(B_{0}\right)$ (Fig. S21B). These variations correspond to the switch of net effect of primary producers on mineral nutrients from being negative to positive (Fig. 5B in the main text). Thus, the positive interaction between the two compartments deceases the dampening of the initial perturbation and decreases the return rate to equilibrium. However, the invariability of each compartment is not very different between $\lambda=0$ and $\lambda=1$ (Fig. 4), which shows that asymptotic resilience is not representative of the stability of each compartment.

\section{Food chain length equal to 2 (TL2)}

Here, we consider a food chain with a food chain length equal to 2 (TL2) where herbivores $B_{2}$ are perturbed but we obtain results similar to those in Fig. 4 in the main text. In fact, herbivores $B_{2}$ are destabilised by nutrient cycling as well as mineral nutrients, which are at even trophic distances from the herbivores (Fig. S22). Moreover, primary producers, which are at odd trophic distances from the herbivores, are stabilised by nutrient cycling except for $D \geq 0.1$, where we observe a maximum of invariability as for mineral nutrients in Fig. 4 in the main text. This result is also explained by the terms of the Jacobian matrix (Fig. S2-3), which are graphically summarised in Fig. 7 in the main text. Single terms such as $J_{01}$ represent direct effects (effects of $B_{1}$ on $B_{0}$ ), while products of terms such as $J_{01} J_{12}$ represent indirect effects (effects of $B_{2}$ on $B_{0}$ ) (Neutel and Thorne, 2014).

$$
\begin{aligned}
J_{01} J_{12} & =-a_{1} B_{1}\left[-a_{1} B_{0}+\lambda\left(m+2 D B_{1}\right)+\lambda\left(1-b_{2}\right) a_{2} B_{2}\right] \\
J_{02} & =\lambda\left(m+2 D B_{2}\right)+\lambda\left(1-b_{2}\right) a_{2} B_{1} \\
J_{10} J_{02} & =\left[\lambda\left(m+2 D B_{2}\right)+\lambda\left(1-b_{2}\right) a_{2} B_{2}\right] a_{1} B_{1} \\
J_{12} & =-a_{2} B_{1}
\end{aligned}
$$

These terms show the variations in the strength of direct and indirect interactions but they cannot be compared directly, as they do not have the same dimension. The maximum stability seen previously for $B_{1}$ is due to the 

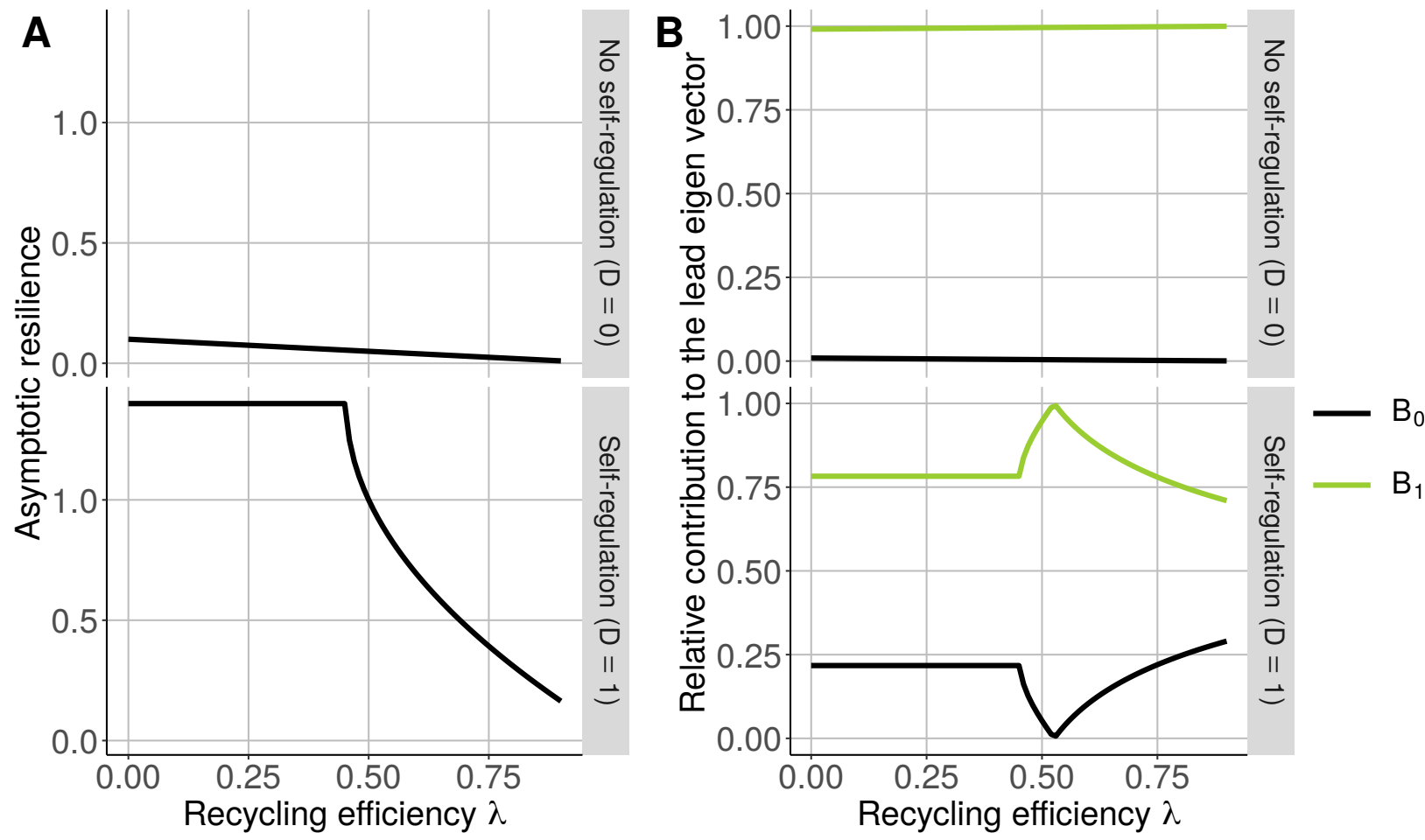

Figure S2-1: Linear stability depending on recycling efficiency $\lambda$ and self-regulation coefficient $D$ in a food chain with a chain length equal to 1 (TL1). A) Asymptotic resilience (opposite of the real part of the lead eigen value of the Jacobian matrix of the system at equilibrium). B) Relative contribution of each compartment to the lead eigen vector.

strong increase in $J_{10} J_{02}$ (indirect positive effect of herbivores on prey mediated by nutrient cycling) with $\lambda$, which offsets the direct negative effect quantified by $J_{12}$ (Fig. S2-3). As in Fig. S2-1A, asymptotic resilience decreases with recycling efficiency $\lambda$ when self-regulation $D$ is high (Fig. S2-4A). The variations of asymptotic resilience are similar to the variation of herbivore invariability (Fig. S2-2 in the main text) because herbivores have the highest contribution to the lead eigenvector (Fig. S2-4B). The maximum contribution of herbivores and minimum contribution of mineral nutrients $(D=0.1$ and $\lambda \simeq 0.75)$ matches with the maximum of invariability of mineral nutrients in Fig. S2-2 in the main text, which must correspond to a net null effect of herbivores on mineral nutrients. Again, asymptotic resilience only reflects the response of the stability of one trophic level and ignores the effects of the other compartments of the ecosystem.

\section{S2-2 Self-regulation}

\section{S2-2-1 Jacobian matrix}

We can explain the increase in the invariability of the mineral nutrients $B_{0}$ based on the terms of the Jacobian matrix mapped in Fig. 7A. With three compartments, we must consider the direct effect of herbivores $B_{2}$ on mineral nutrients $B_{0}$ represented by the term $J_{02}$ and the indirect effect represented by the product of the terms $J_{12} J_{01}$.

In Fig. S2-5, we see that the overall effect of herbivores on mineral nutrients is mostly positive except for $\lambda=1$ where $J_{12} J_{01}$ is negative for a high self-regulation coefficient $D$. However, the direct effect $J_{02}$ must prevail, as the variations in the standard deviation of the nutrients are similar to those of the herbivores (Fig. 6).

In comparison to other values of $\lambda$, the invariability of the primary producers $B_{1}$ for $\lambda=1$ has a different response to self-regulation. The first increase in the invariability at $D=10^{-3}$ is explained by an increase in the biomass of primary producers due to a decrease in the predation of $B_{2}$ on $B_{1}$. In fact, self-regulation affects more herbivores that have a higher biomass than primary producers and releases them from top-down control. The increase in self-regulation increases the positive term $J_{02} J_{10}$ which first compensates for the negative effect of predation $J_{12}$, thus increasing the invariability. Then, the dramatic increase in $J_{02} J_{10}$ should overwhelm $J_{02}$ and perturbations should become transmitted mainly by nutrient cycling, thus leading to a decrease in invariability (Fig. S2-5). 


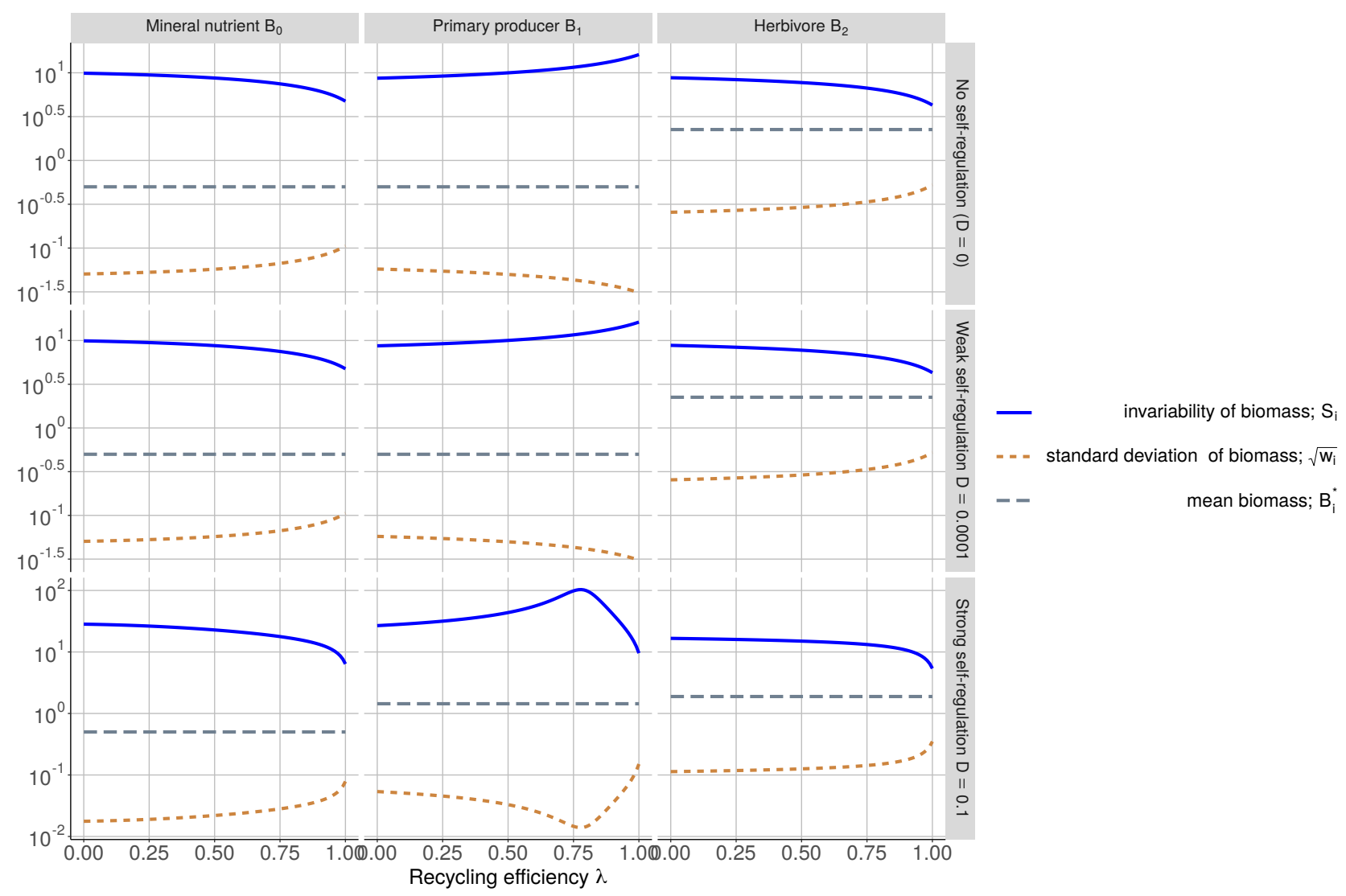

Figure S2-2: Stability of different compartments depending on recycling efficiency $\lambda$ and self-regulation coefficient $D$ in a food chain with a chain length equal to 2 (TL2). Herbivores are perturbed, basal external inputs $I$ are fixed to 0.05 , and predation rate $a_{2}$ is equal to 0.4 when $D=0$ and to 0.401 when $D \neq 0$.

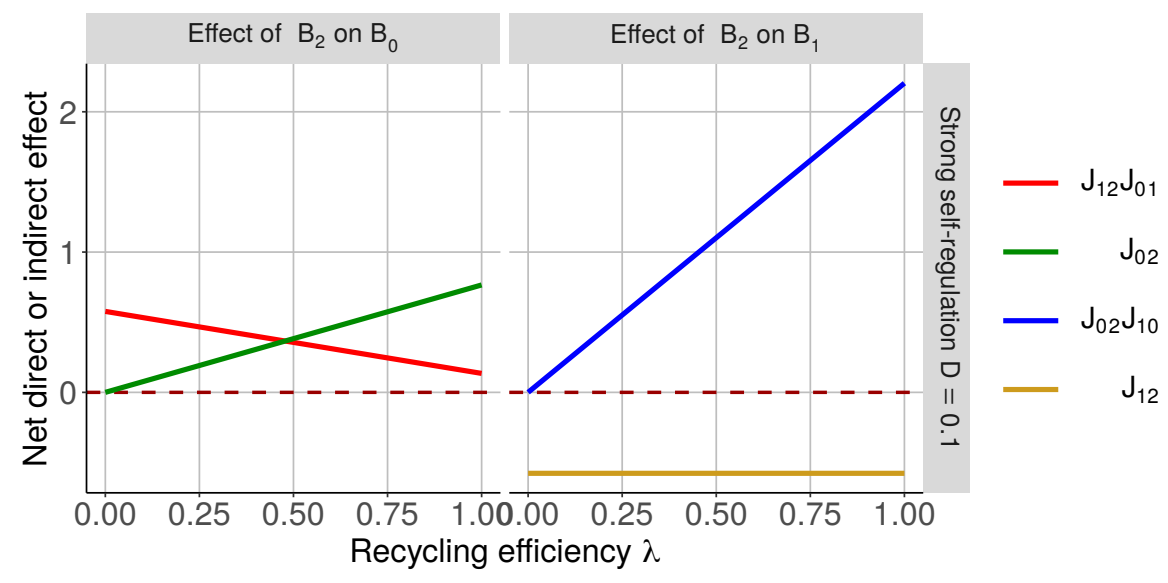

Figure S2-3: Variations in the direct and indirect interaction strengths affecting each species depending on recycling efficiency $\lambda$ and self-regulation coefficient $D$. Interaction strengths affecting mineral nutrients $B_{0}$ and primary producers $B_{1}$ are calculated from the elements $J_{i j}$ of the Jacobian matrix. The dashed red line represents null values. 

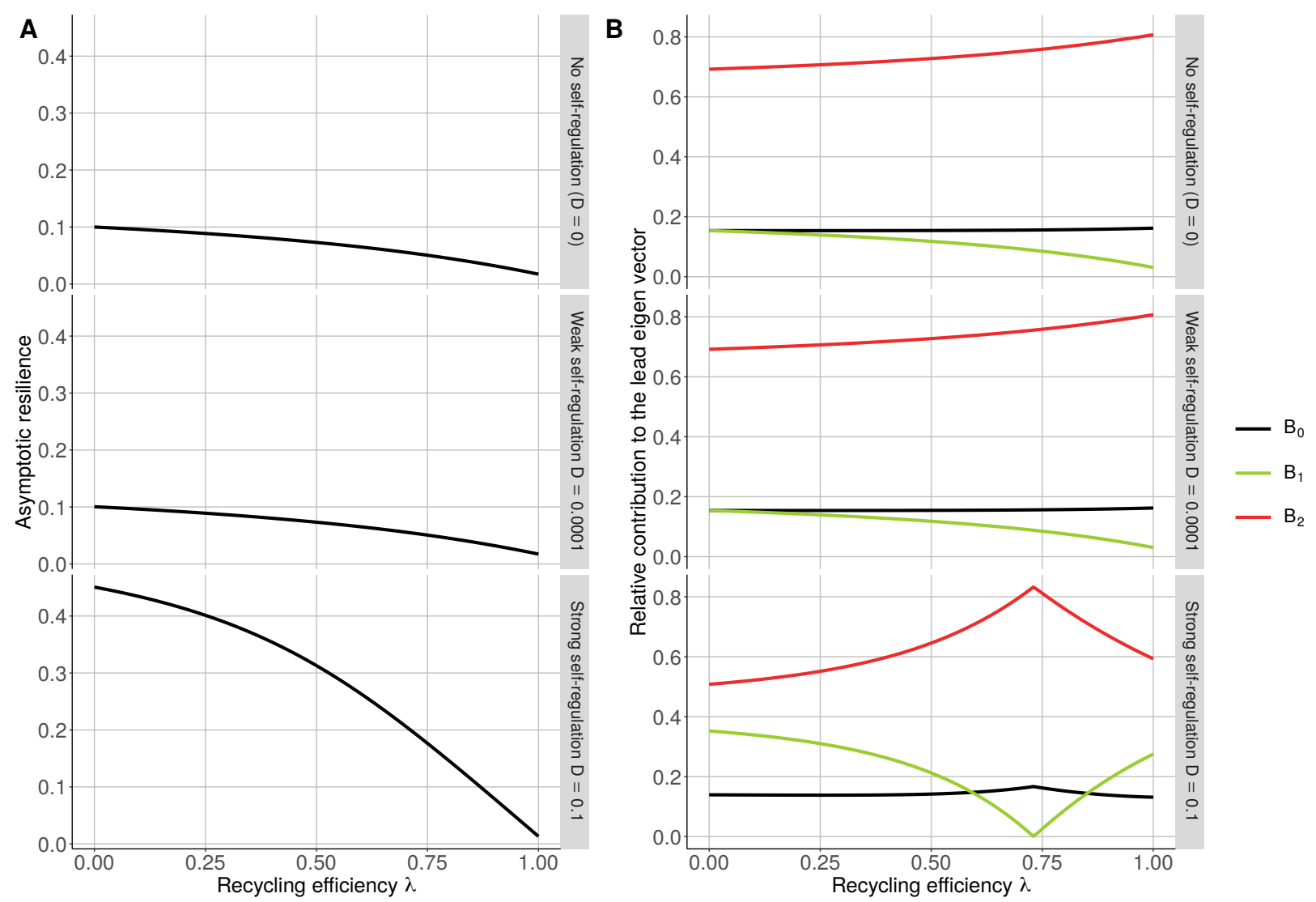

Figure S2-4: Linear stability depending on recycling efficiency $\lambda$ and self-regulation coefficient $D$ in a food chain with a chain length equal to 2 (TL2). A) Asymptotic resilience (opposite of the real part of the lead eigen value of the Jacobian matrix of the system at equilibrium). B) Relative contribution of each compartment to the lead eigen vector. 


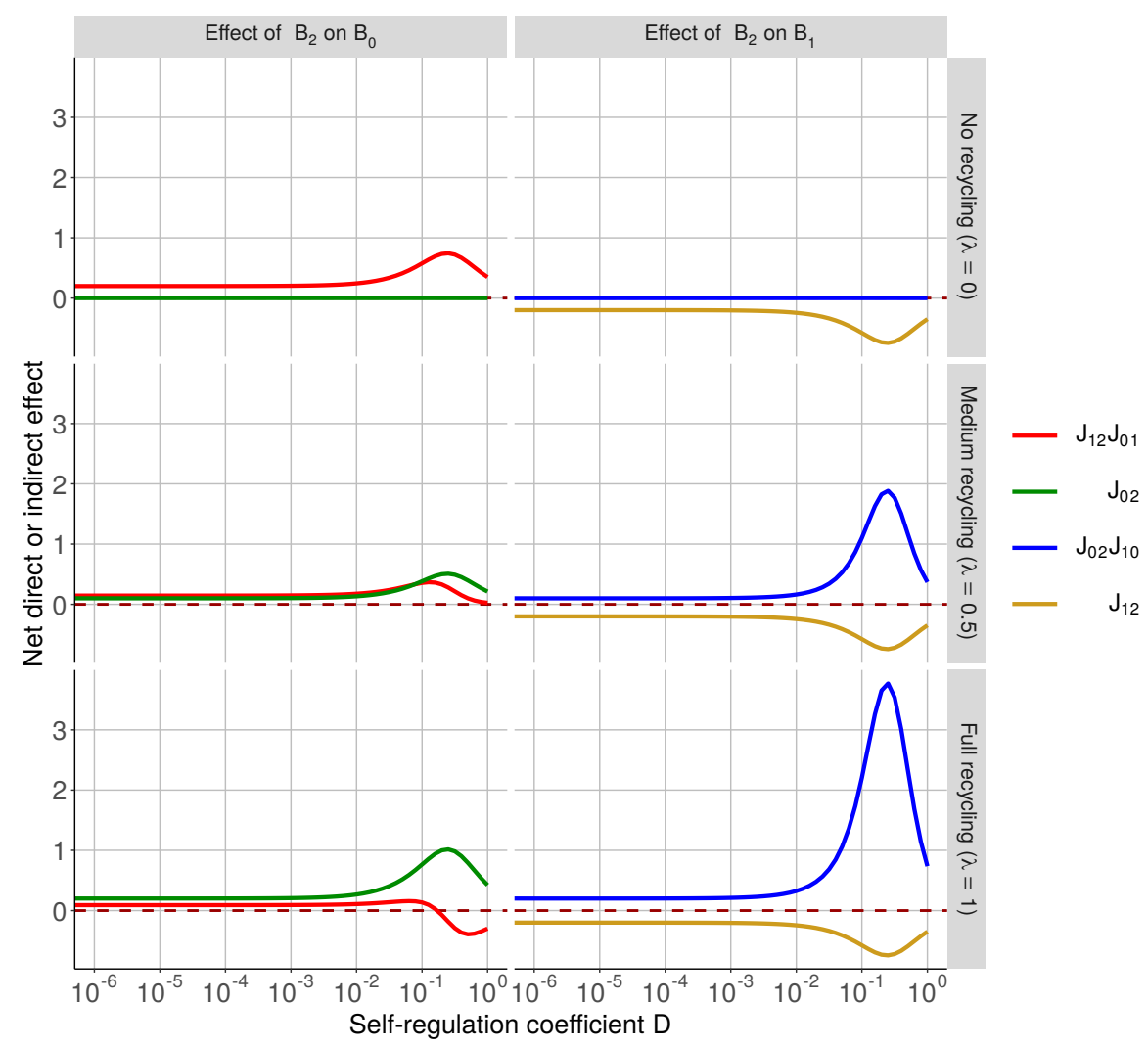

Figure S2-5: Variations in the direct and indirect effects affecting each species depending on recycling efficiency $\lambda$ and self-regulation coefficient $D$. The effects are calculated from the elements of the Jacobian matrix $J$ for mineral nutrients $B_{0}$ and primary producers $B_{1}$. The dashed red line represents the null value of the elements of the Jacobian matrix. $J_{12} J_{01}=-a_{1} B_{1}\left[-a_{1} B_{0}+\lambda\left(m+2 D B_{1}\right)+\lambda\left(1-b_{2}\right) a_{2} B_{2}\right]$; $J_{02}=\lambda\left(m+2 D B_{2}\right)+\lambda\left(1-b_{2}\right) a_{2} B_{1} ; J_{02} J_{10}=\left[\lambda\left(m+2 D B_{2}\right)+\lambda\left(1-b_{2}\right) a_{2} B_{2}\right] a_{1} B_{1} ; J_{12}=-a_{2} B_{1}$. The parameter $a_{2}$ is equal to 0.4 when $D=0$ and to 0.401 when $D \neq 0$.

Finally, the invariability of the primary producers increases again. Self-regulation, which induces additional mortality, decreases the biomass of the primary producers $B_{1}$ and herbivores $B_{2}$, thus leading to a decrease in the quantity of recycled nutrients. This dramatically decreases $J_{02} J_{10}$ and leads to a rebound in the invariability of primary producers $B_{1}$. If self-regulation continues to increase, then the biomass of primary producer will continue to drop and then decrease invariability.

\section{Asymptotic resilience}

Asymptotic resilience has a humped-shaped relationship with self-regulation $D$ when recycling efficiency $\lambda$ is low or intermediate $(\lambda=0$ or $\lambda=0.5)$ with a maximum for $D \simeq 10^{-1}$ (Fig. S2-6A). This humped-shaped relationship can be explained by the transitory decrease of the contribution of herbivores $\left(B_{2}\right)$ and increase of the contribution of primary producers $\left(B_{1}\right)$ to the lead eigenvector (Fig. S2-6B). In fact, primary producer invariability increases with self-regulation $D$ (Fig. 6 in the main text) and this stabilisation is also seen in the asymptotic resilience because of the transitory increased contribution of primary producers. When recycling efficiency is high $(\lambda=1)$, asymptotic resilience is independent of self-regulation $D$, while the invariability of each compartment varies with $D$.

\section{S2-2-2 Food chain length equal to 1 (TL1)}

Here, we show the effects of self-regulation on a food chain with a food chain length equal to 1 (TL1). First, the invariability of the primary producers $B_{1}$ is constant because biomass and standard deviation decrease with self-regulation $D$ at the same rate (Fig. S2-7). In fact, as perturbations have an effect proportional to species biomass (see equation (2) in the main text) we can easily understand that standard deviation varies in the same way as equilibrium biomass.

The invariability of the mineral nutrients $B_{0}$ is constant for all self-regulation values as well as their biomass and 

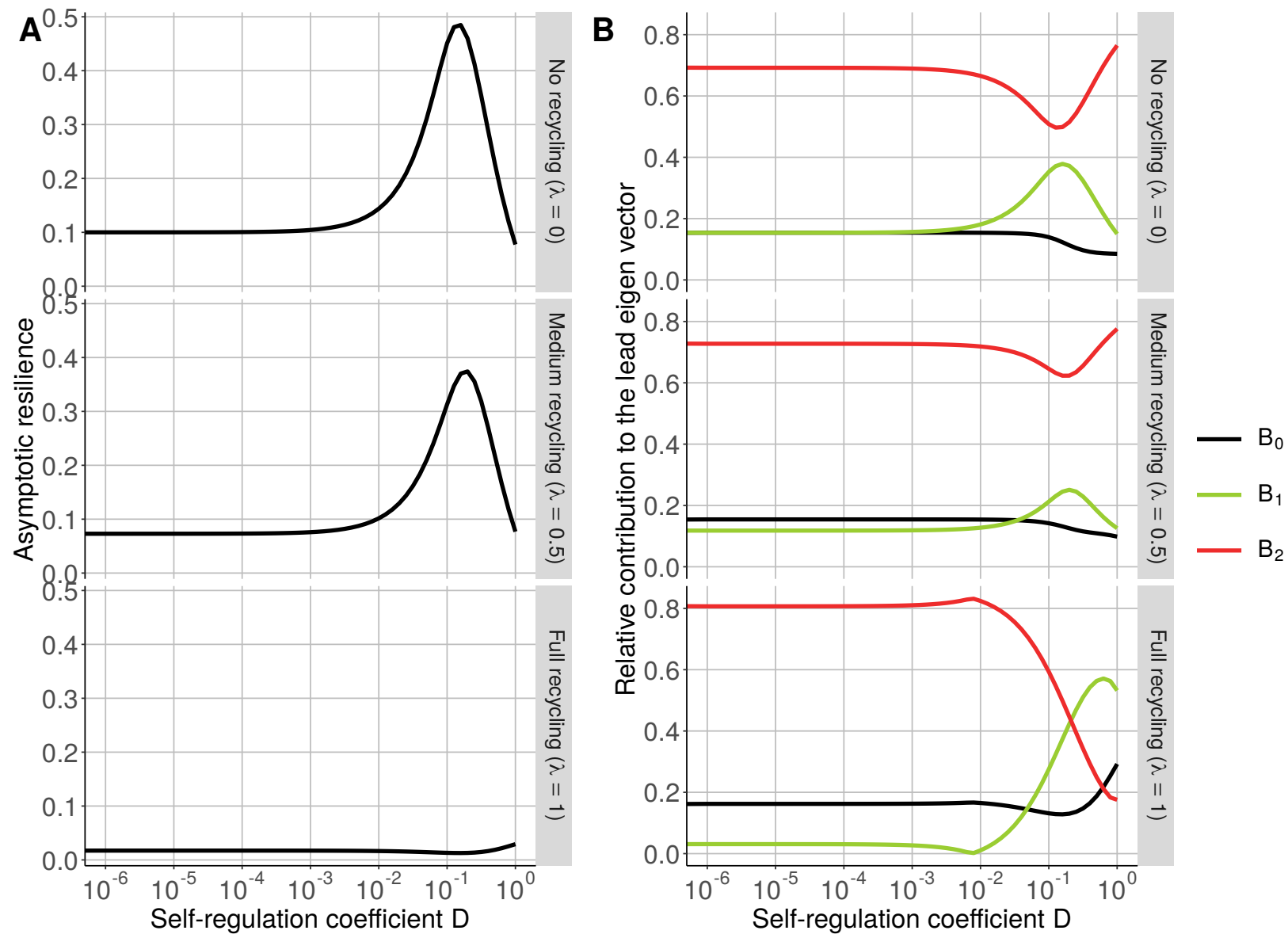

Figure S2-6: Linear stability depending on recycling efficiency $\lambda$ and self-regulation coefficient $D$ in a food chain with a chain length equal to 2 (TL2). A) Asymptotic resilience (opposite of the real part of the lead eigen value of the Jacobian matrix of the system at equilibrium). B) Relative contribution of each compartment to the lead eigen vector. 


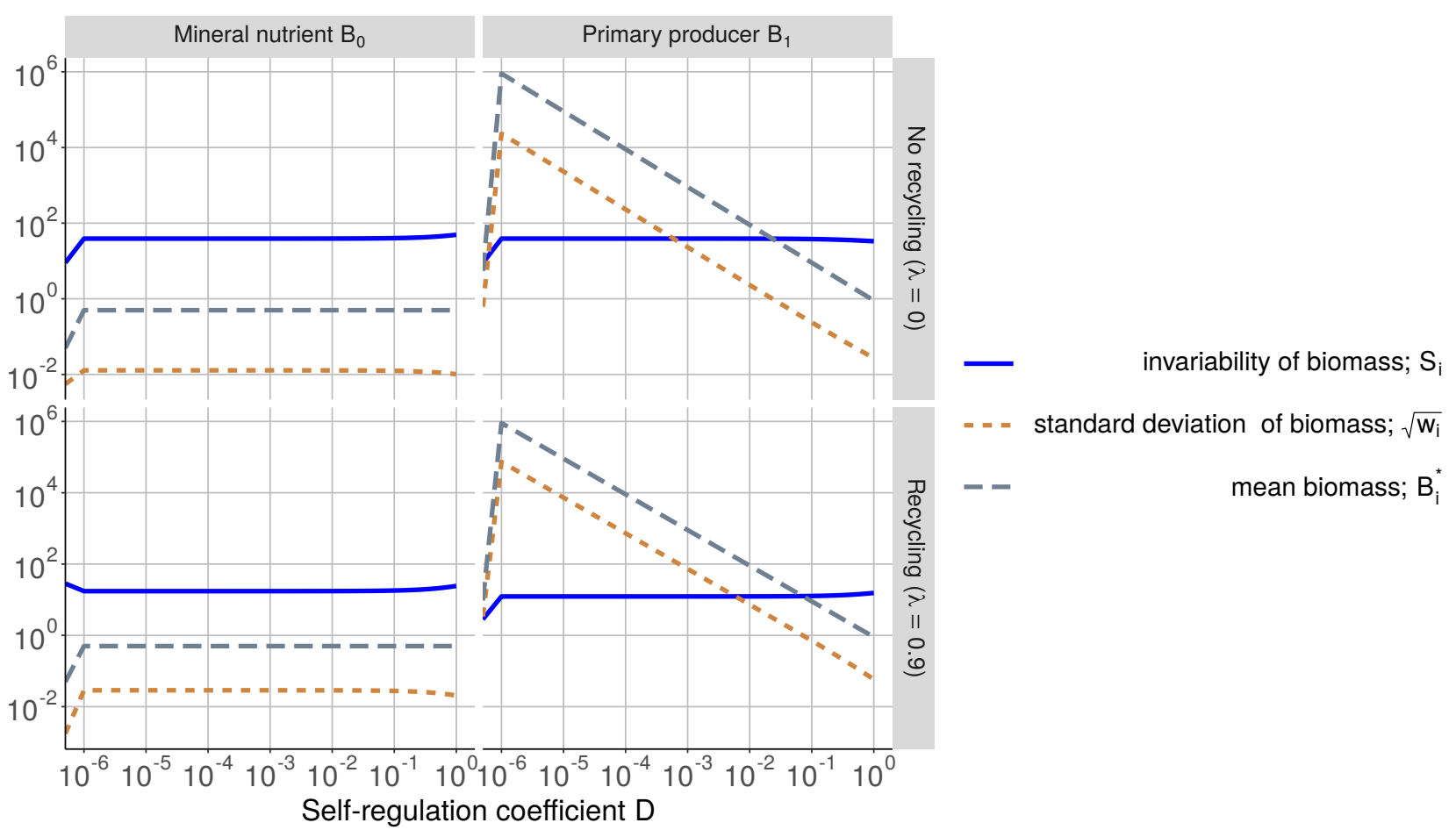

Figure S2-7: Stability of the different compartments depending on the recycling efficiency $\lambda$ and the selfregulation coefficient $D$ in a food chain with a chain length equal to 1 (TL1). Stability is measured by the invariability (i.e., inverse of $\mathrm{CV}$ ), the ratio between equilibrium $B_{i}^{*}$ and the standard deviation $\sigma_{i}$. The parameter $I$ is fixed to 0.05 .

standard deviation (Fig. S2-7). The drop in primary producer biomass affects its positive and negative effects on mineral nutrients in a similar way. In fact, mineral nutrients are negatively affected by uptake by primary producers, which decreases with self-regulation due to the decrease in the biomass of $B_{1}$. Mineral nutrients are also positively affected by the flow of nutrients released by primary producers, which also decreases with self-regulation for the same reasons. Therefore, these two opposite mechanisms have similar variations, thus leading to a constant biomass and standard deviation of mineral nutrients. For $\lambda=0$, the analysis is similar except that the compensation of nutrient cycling lead to the same effect on nutrient stock.

In addition, we notice a discontinuity between the presence or absence of self-regulation $D$, which can be understood analytically. Equation (14) shows the biomass of primary producers at equilibrium $B_{1}^{*}$ for $D>0$ and $\lambda=1$, and when $D$ tends to $0, B_{1}^{*}$ tends to $+\infty$.

$B_{1}^{*}=\frac{I a_{1}-m \ell}{D \ell}$

In a model without self-regulation (i.e., self-regulation is removed from equations and not just set to zero), $B_{0} *$ is defined by:

$B_{0} *=\frac{m}{a_{1}}=\frac{I}{\ell}$

$B_{1}^{*}$ is not defined, equilibrium only exists if the parameter values verify equation (15). If the equation is verified, then $B_{1}^{*}$ is equal to its initial value because the quantity of recycled nutrients is equal to the quantity of nutrients absorbed. For longer chain lengths, we do not see such a discontinuity.

$B_{1}^{*}=\frac{I a_{1} D+a_{2} m \ell-D m \ell}{a_{2}^{2} b_{2} \ell+D^{2} \ell}$

A discontinuity for $D=0$ is also observed in asymptotic resilience (Fig. S2-8A). However, asymptotic resilience varies with self-regulation $D$, while the invariability of each compartment is independent of $D$ (Fig. S2-7).

\section{S2-3 Food chain length}

As in Fig. $8 \mathrm{~A}$ in the main text, the response of the stability of mineral nutrients to the recycling efficiency $\lambda$ is altered by food chan length. Stability increases with $\lambda$ if mineral nutrients are at odd trophic distances from 

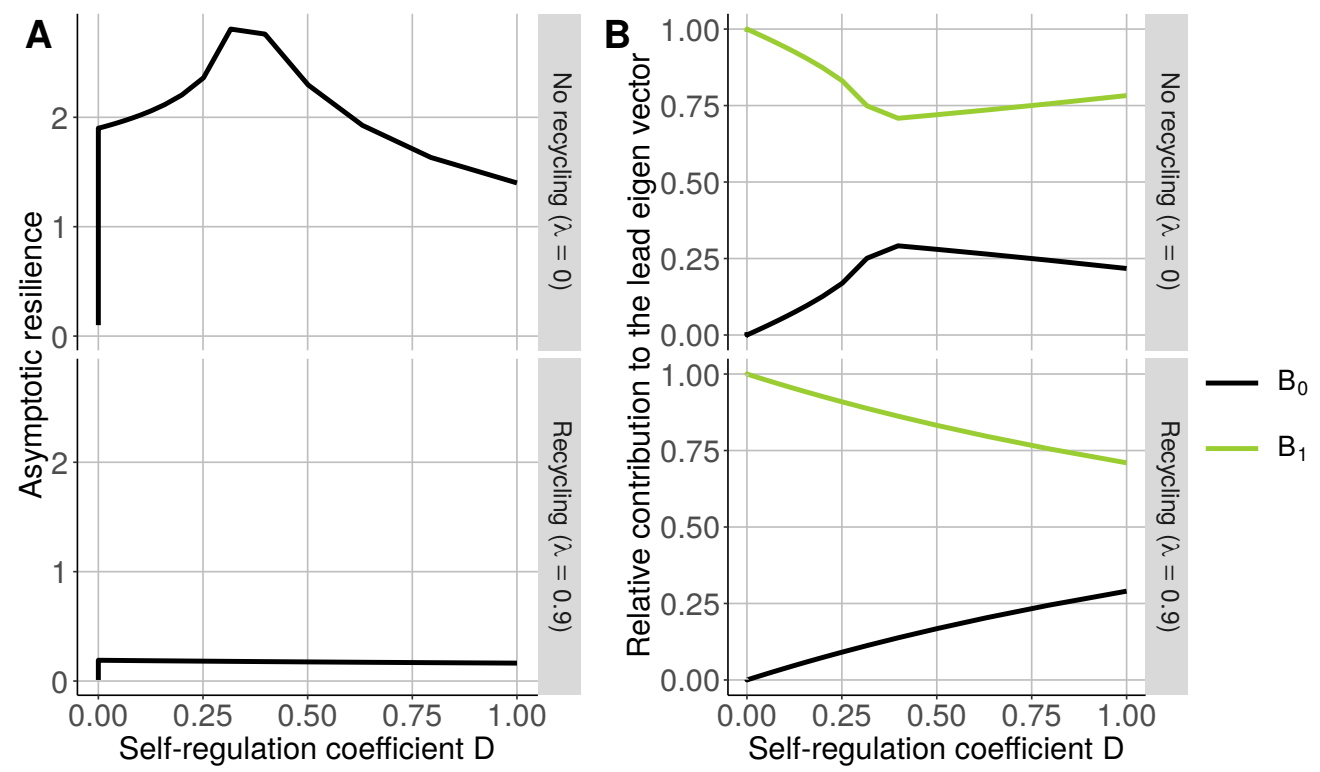

Figure S2-8: Linear stability depending on recycling efficiency $\lambda$ and self-regulation coefficient $D$ in a food chain with a chain length equal to 1 (TL1). A) Asymptotic resilience (opposite of the real part of the lead eigen value of the Jacobian matrix of the system at equilibrium). B) Relative contribution of each compartment to the lead eigen vector.

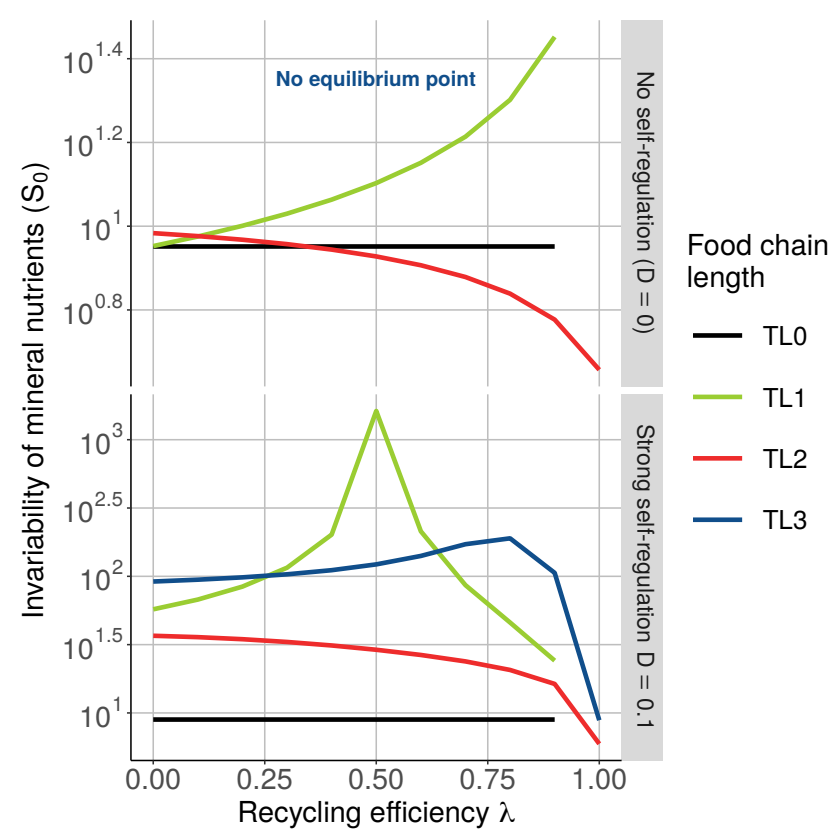

Figure S2-9: Stability of the mineral nutrients $B_{0}$ depending on recycling efficiency $\lambda$ and self-regulation coefficient $D$ for various chain lengths. Stability is measured by invariability (i.e., inverse of CV), the ratio between equilibrium biomass $B_{i}^{*}$ and standard deviation $\sigma_{i}$. External nutrient input $I$ is fixed to 0.1 and attack rate $a_{2}$ is equal to 0.401 . 
the perturbed species (TL1 and TL3) and decreases if they are at even trophic distances (TL2) (Fig. S2-9). When the mineral nutrients are at odd distance, the maximum invariability described in Fig. 4 for TL1 exists for TL3 but is less pronounced. Thus, the mitigation of the negative effect of consumption by nutrient cycling acts at long trophic distances but is dampened.

\section{S2-4 Perturbation of mineral nutrients}

In the main text, we only considered perturbations affecting the top trophic level. When mineral nutrients $B_{0}$ are perturbed, the effect of nutrient cycling is largely diminished (Fig. S2-10A), and we only observed destabilisation when the recycling efficiency $\lambda$ increases. In fact, the bottom-up effect of the perturbation is amplified by the bottom-up effect of nutrient cycling, while opposite effects are shown in Fig. 4 in the main text.

The invariability of the mineral nutrients $B_{0}$ and the primary producers $B_{1}$ decrease with the self-regulation coefficient $D$ because the biomass of the primary producers $B_{1}$ decreases, while its standard deviation remains constant (Fig. S2-10B). Under the effect of self-regulation, the biomass of the primary producers $B_{1}$ decreases, while its standard deviation remains constant. The decrease in the invariability of the mineral nutrients is explained by the increase in the standard deviation due to a decrease in nutrient uptake by $B_{1}$, which diminishes the dampening effect of the primary producers.
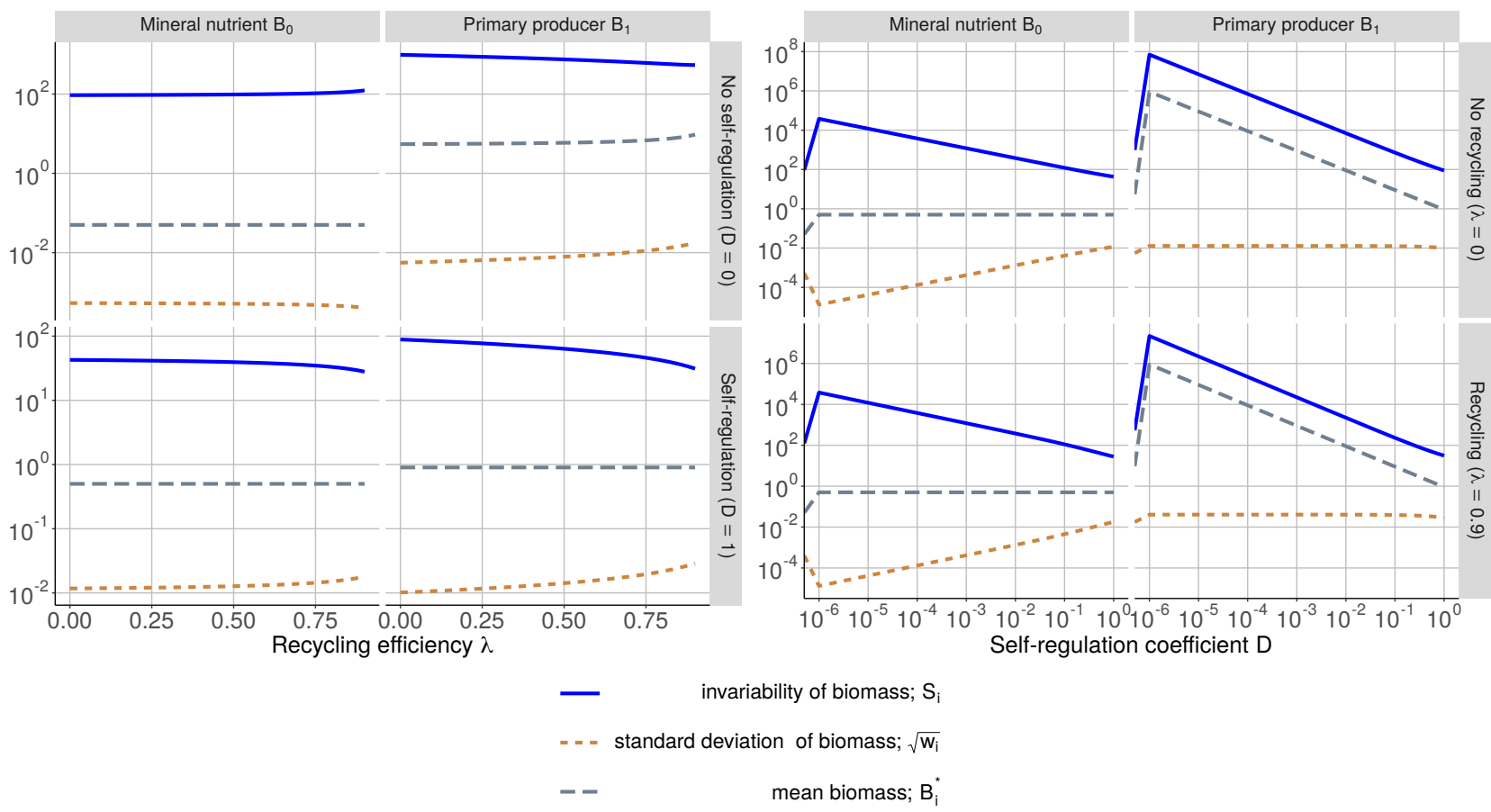

Figure S2-10: Stability of the different compartments depending on A) recycling efficiency $\lambda$ and B) selfregulation coefficient $D$ in a food chain with a chain length equal to 1 (TL1). Stability is measured by invariability (i.e., inverse of $\mathrm{CV}$ ), which is the ratio of equilibrium biomass $B_{i}^{*}$ to standard deviation $\sigma_{i}$. External nutrient input $I$ is fixed to 0.05 .

\section{References}

Arnoldi, J., Loreau, M., \& Haegeman, B. (2016). Resilience, reactivity and variability: A mathematical comparison of ecological stability measures. Journal of Theoretical Biology, 389, 47-59. doi:10.1016/j.jtbi. 2015.10.012

Haegeman, B., Arnoldi, J.-F., Wang, S., de Mazancourt, C., Montoya, J. M., \& Loreau, M. (2016). Resilience, invariability, and ecological stability across levels of organization. bioRxiv. doi:10.1101/085852

McCann, K. S. (2000). The diversity-stability debate. Nature, 405(6783), 228-233. doi:10.1038/35012234

Neutel, A.-M., \& Thorne, M. A. (2014). Interaction strengths in balanced carbon cycles and the absence of a relation between ecosystem complexity and stability. Ecology Letters, 17(6), 651-661. doi:10.1111/ele. 12266 